\title{
A Broad Role for Melanopsin in Nonvisual Photoreception
}

\author{
Joshua J. Gooley, ${ }^{1}$ Jun Lu, ${ }^{1}$ Dietmar Fischer, ${ }^{2}$ and Clifford B. Saper ${ }^{1}$ \\ ${ }^{1}$ Department of Neurology, Beth Israel Deaconess Medical Center, Boston, Massachusetts 02215, and Program in Neuroscience, Harvard Medical School, \\ Boston, Massachusetts 02115, and 'Laboratories in Neuroscience Research in Neurosurgery, Children's Hospital, Boston, Massachusetts 02115
}

The rod and cone photoreceptors that mediate visual phototransduction in mammals are not required for light-induced circadian entrainment, negative masking of locomotor activity, suppression of pineal melatonin, or the pupillary light reflex. The photopigment melanopsin has recently been identified in intrinsically photosensitive retinal ganglion cells (RGCs) that project to the suprachiasmatic nucleus (SCN), intergeniculate leaflet (IGL), and olivary pretectal nucleus, suggesting that melanopsin might influence a variety of irradiance-driven responses. We have found novel projections from RGCs that express melanopsin mRNA to the ventral subparaventricular zone (vSPZ), a region involved in circadian regulation and negative masking, and the sleep-active ventrolateral preoptic nucleus (VLPO) and determined the subsets of melanopsin-expressing RGCs that project to the SCN, the pretectal area (PTA), and the IGL division of the lateral geniculate nucleus (LGN). Melanopsin was expressed in the majority of RGCs that project to the SCN, vSPZ, and VLPO and in a subpopulation of RGCs that innervate the PTA and the IGL but not in RGCs projecting to the dorsal LGN or superior colliculus. Two-thirds of RGCs containing melanopsin transcript projected to each of the SCN and contralateral PTA, and one-fifth projected to the ipsilateral IGL. Double-retrograde tracing from the SCN and PTA demonstrated a subpopulation of RGCs projecting to both sites, most of which contained melanopsin mRNA. Our results suggest that melanopsin expression defines a subset of RGCs that play a broad role in the regulation of nonvisual photoreception, providing collateralized projections that contribute to circadian entrainment, negative masking, the regulation of sleep-wake states, and the pupillary light reflex.

Key words: melanopsin; ganglion cells; circadian; entrainment; sleep; pupillary light reflex; masking; retina; suprachiasmatic; subparaventricular; ventrolateral preoptic nucleus; pretectal; intergeniculate; hypothalamus; adeno-associated virus

\section{Introduction}

Mammalian circadian rhythms are generated endogenously in the suprachiasmatic nucleus (SCN) in the anterior hypothalamus. The retinohypothalamic tract conveys photic information to the SCN, synchronizing the circadian pacemaker to the $24 \mathrm{hr}$ solar cycle. Transgenic mice lacking rods and cones are functionally blind but retain the ability to entrain to an imposed light/dark cycle, suggesting that a nonvisual photic system is capable of mediating circadian entrainment (Freedman et al., 1999). Recently, the novel photopigment melanopsin was discovered in the mammalian inner retina (Provencio et al., 2000, 2002). We showed that most retinal ganglion cells (RGCs) that contain melanopsin mRNA project to the SCN (Gooley et al., 2001). Conversely, most RGCs that project to the SCN also contain melanopsin transcript. The presence of melanopsin in RGCs that project to the SCN was independently demonstrated by Hannibal et al. (2002), who showed that melanopsin is present in pituitary adenylate cyclase-activating polypeptide-containing RGCs, which contribute to the retinohypothalamic tract. Melanopsin is

\footnotetext{
Received 0ct. 15, 2002; revised April 16, 2003; accepted May 12, 2003.

This work was supported by United States Public Health Service Grants HL60292, HL07901, and MH67413, and the German Research Foundation (DFG). We thank Quan Ha and Minh Ha for superb technical assistance, and Dr. Thomas Scammell and Thomas Chou for helpful discussions and suggestions concerning experiments described in this manuscript.

Correspondence should be addressed to Dr. Clifford B. Saper, Department of Neurology, Beth Israel Deaconess Medical Center, 330 Brookline Avenue, Boston, MA 02215. E-mail: csaper@caregroup.harvard.edu. Copyright $\odot 2003$ Society for Neuroscience $\quad$ 0270-6474/03/237093-14\$15.00/0
}

therefore a primary candidate photopigment for mediating the synchronization of endogenous circadian rhythms to the light/ dark cycle.

Remarkably, RGCs that contain melanopsin are directly photosensitive and depolarize in response to light (Berson et al., 2002; Hattar et al., 2002). In heterozygous mice with a targeted disruption of the melanopsin (Opn4) gene and an added tau-LacZ reporter gene, $\beta$-galactosidase activity was detected in axons projecting to the SCN, intergeniculate leaflet (IGL), and olivary pretectal nucleus (OPT) (Hattar et al., 2002). These results suggest that melanopsin may be involved in both the regulation of circadian rhythms and pupillary light reflex. Recent studies in $O p n 4^{-1-}$ mice have shown that melanopsin is required for normal circadian phase resetting (Panda et al., 2002; Ruby et al., 2002) and pupillomotor constriction in response to bright light (Lucas et al., 2003). However, these studies leave several important questions unanswered. The distribution and proportion of melanopsin-positive RGCs that project to each brain target remains unknown. In addition, nonclassical photoreceptors have been implicated in other light-mediated responses, such as the regulation of melatonin secretion, negative masking, and adaptation of the human primary cone visual pathway (Freedman et al., 1999; Lucas et al., 1999, 2001; Brainard et al., 2001; Mrosovsky et al., 2001; Thapan et al., 2001; Hankins and Lucas, 2002). It is therefore possible that melanopsin influences a variety of irradiance-driven behaviors. However, it is not known whether melanopsin-containing RGCs project to other retinorecipient 
brain regions such as the subparaventricular zone (SPZ) (Levine et al., 1991), which is implicated in negative masking (Kramer et al., 2001), or the ventrolateral preoptic nucleus (VLPO), which is involved in sleep regulation (Sherin et al., 1996, 1998; Lu et al., 1999, 2000, 2002). Furthermore, it is not known whether the projections to the SCN, IGL, and pretectal area (PTA) are attributable to branching of axons from a single population of melanopsin-positive RGCs, or whether the different sites receive unique inputs.

To address these issues, we first used a novel method of tracing the projections of melanopsin-containing RGCs with recombinant adeno-associated virus containing a green fluorescent protein (GFP) reporter gene [rAAV-GFP (derived from AAV-2 serotype, with a constitutively active cytomegalovirus- $\beta$-globin hybrid promoter) (Harvard Gene Therapy Initiative, Boston, MA)], which preferentially transduces melanopsin-containing RGCs. We then systematically injected retinal targets, including the SCN, ventral SPZ (vSPZ), VLPO, lateral geniculate nucleus (LGN), PTA, and superior colliculus (SC), with retrograde tracer and examined retrogradely labeled RGCs for melanopsin mRNA. Double-retrograde fluorescent labeling was used to detect axonal collateralization to the SCN and pretectal area.

\section{Materials and Methods}

Animals. Adult male Sprague Dawley rats (275-300 gm) (Harlan Sprague Dawley, Indianapolis, IA) were maintained in a 12:12 light/dark cycle with food and water provided ad libitum (lights on at 7:00 A.M.). All protocols were approved by the Institutional Animal Care and Use Committees of Beth Israel Deaconess Medical Center and Harvard Medical School.

Eye injections. To anterogradely label retinal efferents, cholera toxin $\mathrm{B}$ subunit (CTB; List Biologic, Campbell, CA) or rAAV-GFP was injected unilaterally or bilaterally into the vitreous body of the eye. Rats were anesthetized with $7 \%$ chloral hydrate ( $350 \mathrm{mg} / \mathrm{kg}$, i.p.), and a micro knife (Xomed Surgical Products, Jacksonville, FL) was used to puncture the eye near the cornea-scleral junction. A $10 \mu \mathrm{l}$ Hamilton syringe was then inserted in the puncture hole and aimed behind the lens into the vitreous chamber, in which $5 \mu \mathrm{l}$ of $1 \%$ CTB or $4 \mu \mathrm{l}$ of rAAV-GFP $\left(6.8 \times 10^{9}\right.$ viral particles) were injected. After the injection, the needle remained in the eye for $10 \mathrm{sec}$ before being slowly withdrawn. For larger injections of rAAV-GFP, a posterior injection approach was used as described previously (Fischer et al., 2001). Briefly, a midline incision was made in the scalp, and the skin was peeled back to expose the dorsal aspect of the eye. Part of the lacrimal gland and dorsal extraocular muscles were then surgically removed to allow direct access to the optic nerve head. A glass micropipette was used to puncture the cornea, and $\sim 10 \mu \mathrm{l}$ of aqueous fluid were drawn from the anterior chamber. A second glass micropipette was then used to pierce the retina immediately dorsal to the optic disc, in which $10 \mu \mathrm{l}$ of rAAV-GFP $\left(1.7 \times 10^{10}\right.$ viral particles $)$ were injected into the vitreous body. Animals injected with CTB were individually housed for 1 week before being killed, and rAAV-GFP-injected rats were killed after 2-3 weeks.

Brain injections. To retrogradely label retinal projections to the brain, animals were anesthetized with $7 \%$ chloral hydrate $(350 \mathrm{mg} / \mathrm{kg}$, i.p.) and placed in a stereotaxis for surgery. After an incision of the scalp, a burr hole was made above the injection site. A glass micropipette containing either 5\% FluoroGold (FG; Fluorochrome, Inc., Englewood, CO) or 1\% $\mathrm{CTB}$ was lowered into the desired injection site. Tracer was injected using a compressed air delivery system as described previously (Elmquist and Saper, 1996). The following coordinates in the anteroposterior (AP), dorsoventral (DV), and mediolateral (ML) axes relative to bregma were used (Paxinos and Watson, 1997): SCN, -1.1 AP, -8.9 DV, 2.6 ML (injection was made at $16^{\circ}$ from the vertical axis); vSPZ, $-1.4 \mathrm{AP},-8.5$ $\mathrm{DV}, 2.6 \mathrm{ML}$ (injection was made at $16^{\circ}$ from the vertical axis); VLPO, $-0.4 \mathrm{AP},-8.2 \mathrm{DV},-0.9 \mathrm{ML}$; OPT, $-4.8 \mathrm{AP},-4 \mathrm{DV},-1.2 \mathrm{ML}$; LGN, $-4.5 \mathrm{AP},-4.1 \mathrm{DV},-3.6 \mathrm{ML}$; SC, $-5.8 \mathrm{AP},-3.4 \mathrm{DV},-1 \mathrm{ML}$. Three nanoliters of FG were injected into the SCN or VLPO, $1.5 \mathrm{nl}$ of a mixture of $12.5 \%$ biotinylated dextran amine (BDA) and $1 \%$ CTB were injected into the vSPZ, and $6-9 \mathrm{nl}$ of FG or CTB were injected into the OPT, LGN, or SC. In animals that received both tracers, FG and CTB were administered on the same side of the brain. After an injection, the micropipette was slowly withdrawn, and the surgical site was closed with sterile wound clips. Animals were then individually housed under the aforementioned controlled conditions for 1 week before being killed.

Perfusion and tissue preparation. Rats were deeply anesthetized with $7 \%$ chloral hydrate $(500 \mathrm{mg} / \mathrm{kg}$, i.p. $)$ and transcardially perfused with $0.9 \%$ saline in $0.1 \%$ diethylpyrocarbonate-treated water $\left(\right.$ DEPC $\left.-\mathrm{H}_{2} \mathrm{O}\right)$, followed by $10 \%$ neutral buffered formalin $(500 \mathrm{ml})$. Eyes and brains were removed and postfixed in $10 \%$ formalin for $4 \mathrm{hr}$ and then cryoprotected in $20 \%$ sucrose in DEPC- $\mathrm{H}_{2} \mathrm{O}$. Coronal sections $(40 \mu \mathrm{m})$ through the injection site were cut on a freezing microtome and stored in $0.02 \%$ azide in PBS until mounted on slides or used for immunohistochemistry.

Immunohistochemistry. Immunohistochemistry was performed to verify the FG and CTB injection sites and label retinal projections to the brain in animals that received intravitreal injections of either CTB or rAAV-GFP. Free-floating brain sections were washed in PBS, treated with $0.3 \%$ hydrogen peroxide in PBT ( $0.25 \%$ Triton X-100 in PBS) for 30 min, and blocked in 1\% bovine albumin in PBT (BSA-PBT) for $1 \mathrm{hr}$. After additional washes, sections were incubated overnight with primary antiserum [rabbit anti-FG, 1:20,000 (Chemicon, Temecula, CA); goat anti-CTB, 1:100,000 (List Biologic); rabbit anti-GFP, 1:20,000 (Molecular Probes, Eugene, OR)] in BSA-PBT. The following day, sections were washed in PBS and incubated for $1 \mathrm{hr}$ in biotinylated secondary antiserum (1:1000; Jackson ImmunoResearch, West Grove, PA) in BSAPBT. Tissue was washed again in PBS, incubated in ABC (1:1000; Vector Laboratories, Burlingame, CA) in PBS for $1 \mathrm{hr}$, and washed in PBS. Sections were then incubated in $0.06 \%$ 3,3-diaminobenzidine tetrahydrochloride (DAB; Sigma, St. Louis, MO) with $0.01 \%$ hydrogen peroxide in PBS for $\sim 5 \mathrm{~min}$, washed in PBS, and mounted on gelatin-coated glass slides. All incubations and washes were performed at room temperature. For some sections, the brown $\mathrm{DAB}$ reaction product was enhanced by the addition of $0.01 \% \mathrm{NiSO}_{4}$ and $0.05 \% \mathrm{CoCl}$ to produce a black reaction product. For other sections, a silver intensification protocol was used to amplify the DAB reaction product as described previously (Kitt, 1988). Briefly, sections were rehydrated in $\mathrm{H}_{2} \mathrm{O}$ and incubated at $56^{\circ} \mathrm{C}$ for $1 \mathrm{hr}$ in a saturated solution of $1 \%$ silver nitrate neutralized with ammonium hydroxide. Sections were rinsed with $\mathrm{H}_{2} \mathrm{O}$ and incubated at room temperature for $10 \mathrm{~min}$ in $0.1 \%$ gold chloride. After another wash in $\mathrm{H}_{2} \mathrm{O}$, sections were immersed in 5\% sodium thiosulfate for $5 \mathrm{~min}$ at room temperature and rinsed in $\mathrm{H}_{2} \mathrm{O}$ for 20 min. For double labeling of CTB and vasoactive intestinal polypeptide (VIP), sections were immunohistochemically stained for CTB as described above using DAB with $0.01 \%$ $\mathrm{NiSO}_{4}, 0.05 \% \mathrm{CoCl}$, and $0.02 \% \mathrm{H}_{2} \mathrm{O}_{2}$ in $\mathrm{PBS}$ to produce a black reaction product. After several washes in PBS, sections were incubated overnight in rabbit anti-VIP IgG (1:50,000; Chemicon) and were immunohistochemically stained as described previously with DAB to produce a brown reaction product. Finally, sections were counterstained, dehydrated in an ethanol series, cleared in xylenes, and coverslipped.

Fluorescence immunohistochemistry. After the verification of accurate brain injections, eyes were cut into $20-25 \mu \mathrm{m}$ sections on a cryostat and mounted on superfrost slides (Fisher Scientific, Pittsburgh, PA). Sections were rinsed with $0.1 \%$ DEPC-treated PBS (DEPC-PBS) and blocked in BSA-PBT for $1 \mathrm{hr}$. Tissue was then incubated overnight in primary antiserum [FG, 1:3000; CTB, 1:20,000; melanopsin, 1:500 (generous gift from Dr. King-Wai Yau, John Hopkins University School of Medicine, Baltimore, MD)] in BSA-PBT. The following day, sections were rinsed in PBT and incubated for $1 \mathrm{hr}$ in biotinylated secondary antiserum (1:1000; Jackson ImmunoResearch) in BSA-PBT. After a rinse in DEPC-PBS, sections were incubated for $1 \mathrm{hr}$ in Cy3-conjugated streptavidin (1:1000; Jackson ImmunoResearch) in DEPC-PBS. After a final wash in DEPCPBS, sections were air-dried and stored at $4^{\circ} \mathrm{C}$ until additional processing. For double labeling of FG and CTB, sections were incubated overnight with rabbit anti-FG antibody and goat anti-CTB IgG in BSA-PBT (FG, 1:3000; CTB, 1:20,000). The following day, sections were rinsed with DEPC-PBS, followed by a $1 \mathrm{hr}$ incubation with Cy3-conjugated donkey anti-rabbit IgG (1:1000; Jackson ImmunoResearch) and biotin- 
ylated donkey anti-goat antiserum (1:1000; Jackson ImmunoResearch) in BSA-PBT. Sections were then washed in PBS and incubated for $1 \mathrm{hr}$ in Alexa-Fluor 488-conjugated streptavidin (1:1000; Molecular Probes) in DEPC-PBS. For double labeling of GFP and melanopsin, sections were incubated overnight with goat anti-GFP antibody and rabbit antimelanopsin IgG in PBT [GFP, 1:3000 (Rockland Immunochemicals, Gilbertsville, PA); melanopsin, 1:500 (Dr. King Wai-Yau)]. The following day, sections were washed in PBS and incubated for $1 \mathrm{hr}$ with Alexa-Fluor 488-conjugated donkey anti-goat IgG (1:500; Molecular Probes) and biotinylated donkey anti-rabbit antiserum (1:500; Jackson ImmunoResearch) in PBT. Sections were then washed in PBS and incubated for $1 \mathrm{hr}$ in Cy3-conjugated streptavidin in PBS. All incubations and washes were performed at room temperature. For control sections, primary antiserum was omitted.

In situ hybridization. A plasmid containing a 957 bp mouse melanopsin cDNA fragment was used to generate ${ }^{35}$ S-labeled (NEN, Boston, MA) antisense or sense control riboprobes as described previously (Provencio et al., 2000). Immunohistochemically stained eye sections were postfixed in $4 \%$ formalin for $20 \mathrm{~min}$ at $4^{\circ} \mathrm{C}$. Tissue was then dehydrated by a graded alcohol series, delipidated in xylenes, and rehydrated. Sections were immersed in sodium citrate buffer, $\mathrm{pH} 6.0$, and heated until nearly boiling for $10 \mathrm{~min}$. Afterward, tissue was dehydrated by a graded alcohol series and allowed to dry. Antisense or sense riboprobes $\left(10^{6} \mathrm{cpm}\right)$ were added to hybridization buffer [ $1.2 \mathrm{M} \mathrm{NaCl}, 20 \mathrm{~mm}$ Tris, $\mathrm{pH}$ 7.5, 2 mм EDTA, $\mathrm{pH}$ 8.0, $0.02 \%$ sheared ssDNA (Sigma), $0.02 \%$ yeast tRNA (Invitrogen, Grand Island, NY), $0.1 \%$ total yeast RNA (Sigma), $2 \times$ Denhardt's solution, $20 \%$ dextran sulfate in sterile $\mathrm{H}_{2} \mathrm{O}$ ]. The hybridization mixture was heat denatured at $90^{\circ} \mathrm{C}$ for $5 \mathrm{~min}$ and cooled on ice for an additional 5 min. Each slide was covered with $120 \mu \mathrm{l}$ of hybridization mixture, coverslipped, and incubated overnight at $57^{\circ} \mathrm{C}$. The following day, coverslips were removed in $2 \times$ SSC. Sections were then treated with $0.02 \%$ RNase A in RNase buffer for $30 \mathrm{~min}$ at $37^{\circ} \mathrm{C}$, followed by an incubation in RNase buffer for $30 \mathrm{~min}$ at room temperature. Sections were then submitted to high-stringency washes in $2 \times \mathrm{SSC}$ for $1 \mathrm{hr}$ at $50^{\circ} \mathrm{C}, 0.2 \times \mathrm{SSC}$ for $1 \mathrm{hr}$ at $55^{\circ} \mathrm{C}$, and $0.2 \times \mathrm{SSC}$ for $1 \mathrm{hr}$ at $60^{\circ} \mathrm{C}$, followed by rapid dehydration in 0.3 $\mathrm{M}$ ammonium acetate in a graded alcohol series. The tissue was air-dried, dipped in NTB-2 emulsion, and developed using Kodak (Rochester, NY) Dektol developer after a 2 week incubation at $4^{\circ} \mathrm{C}$.

Cell counting in the retina. Because retinal efferents predominantly innervate the contralateral side of the brain, FG- and/or CTBimmunoreactive RGCs and melanopsin mRNA-positive cells were examined in the eye contralateral to the injection site. For SCN- and IGLinjected animals, the ipsilateral eye was also examined, because each SCN and IGL receives nearly equal bilateral input from each retina. In each SCN-injected animal $(n=4)$, five evenly spaced sections ( $320 \mu \mathrm{m}$ apart) through the midpoint of the globe were used for the data analysis. In each section, all retrogradely labeled RGCs were counted. Because of the paucity of retinal projections to the VLPO, retrogradely labeled RGCs were counted in every section through the globe for VLPO-injected animals. Similarly, because afferents to the vSPZ from the retina are sparse, every other section through the eye was analyzed. In PTA-injected $(n=4)$, LGN-injected $(n=5)$, SC-injected $(n=3)$, and SCN-PTA doubleinjected $(n=4)$ animals, five evenly spaced sections (600-900 $\mu \mathrm{m}$ apart) were examined. RGCs were considered positive for melanopsin mRNA if they contained at least three times the number of silver grains per unit area as the background of the retinal ganglion cell layer.

To correct for the overestimation of retrogradely labeled cells that results from simple profile-based counts, cell numbers were adjusted using the Abercrombie (1946) method. Final cell number estimates were determined by the formula $N=n[T /(T+H)]$, where $N$ is the adjusted cell number, $n$ is the observed profile-based count, $T$ is the mean tissue thickness, and $H$ is the mean height of the soma (Geuna, 2000). On the basis of the assumption that RGC cell bodies are approximately spherical, $H$ was measured parallel to the plane of the section. The SEM was calculated for all cell counts. Stereological sampling was not practical both because retrogradely labeled neurons from some sites were rare, and in situ labeling is not uniform through the depth of a section.

Analysis. In animals that received injections of CTB in the eyes, brains were stained immunohistochemically (as described previously) to label the retinal efferents. Selected retinorecipient areas of the brain were mapped and drawn using a light microscope with a camera lucida attachment (Leitz, Wetzlar, Germany). Each injection site was similarly drawn and then summarized as a template. Drawings were scanned and digitized and then redrawn using Canvas software (Deneba Systems, Miami, FL). Photomicrographs were taken with a color digital camera (Kodak DCS460), and images were adjusted for contrast, brightness, and sharpness using Adobe Photoshop (Adobe Systems, Mountain View, CA). Retrogradely labeled RGCs were counted using an epifluorescence microscope with a $20 \times$ objective, and melanopsin mRNA-positive cells were viewed under dark-field illumination.

\section{Results}

Anterograde tracing of projections of melanopsin-containing RGCs by using rAAV-GFP

Previous studies have shown that intravitreal injection of rAAV results in the transduction of RGCs (Grant et al., 1997; Ali et al., 1998; Dreyer et al., 1999; Dudus et al., 1999; Guy et al., 1999; Auricchio et al., 2001; Liang et al., 2001; Harvey et al., 2002; Martin et al., 2002). Because GFP robustly labels cell bodies, axons, and axonal terminals, rAAV-GFP has been used to trace retinofugal projections anterogradely (Dudus et al., 1999). While testing the efficacy of rAAV-GFP to transduce RGCs and trace RGC projections, we fortuitously discovered that GFP was preferentially expressed in RGCs that contain melanopsin (Fig. $1 A, B)$. Similar to previous findings (Dudus et al., 1999), in animals that we injected with large amounts of rAAV-GFP $(n=13$; $10 \mu \mathrm{l} ; 1.7 \times 10^{10}$ viral particles), GFP-immunoreactive axon terminals were found in all retinorecipient targets including the dorsal lateral geniculate (DLG) and ventral lateral geniculate (VLG) nuclei, the SC, and the SCN. However, we found that in animals injected with smaller amounts of $\mathrm{rAAV}-\mathrm{GFP}(n=9 ; 4 \mu \mathrm{l}$; $6.8 \times 10^{9}$ viral particles), GFP-immunoreactive axon terminals were found predominantly in the SCN, IGL, and OPT, which correspond to brain regions that receive input from melanopsincontaining RGCs (Hattar et al., 2002). For example, in animal 2472 (Fig. 1), 81.4\% of GFP-immunoreactive RGCs were also melanopsin-immunoreactive, whereas $46.9 \%$ of melanopsincontaining cells contained GFP. A similar pattern of labeling was observed in animals 2716, 2729, and 2730, in which 78.9, 61.4, and $68.5 \%$ of GFP-immunoreactive cells contained melanopsin, and $47.1,40.1$, and $34.3 \%$ of melanopsin-immunoreactive RGCs expressed GFP. As expected, the most intense sites of innervation by GFP-labeled axons were the SCN, OPT, and IGL (Fig. 1 D, J,L). In comparison, the DLG, VLG, and SC, which are innervated by the retina at least as heavily as the IGL in cholera toxin ( $\beta$ subunit) experiments (Fig. $1 K, M$ ), each showed relatively sparse innervation by GFP-immunoreactive neurons (Fig. $1 L, N$ ). However, GFP-immunoreactive axons in the VLPO and vSPZ (see description below) were nearly as dense as those seen after CTB injections into the vitreous body (Fig. $1 E--H$ ), suggesting that many of these inputs also arise from melanopsin-expressing RGCs. GFP-containing RGCs projected bilaterally and approximately equally to the SCN (Fig. $1 D$ ) but projected predominantly to the contralateral vSPZ, VLPO, and OPT. Although the projection from GFP-producing RGCs to the rostral and midportion of the IGL appeared to be equal and bilateral, the projection to the caudal IGL, located lateral and ventral to the medial geniculate nucleus, was predominantly contralateral.

It is possible that GFP-positive projections to any one of these sites might be predominantly from the $\sim 20-30 \%$ of RGCs that are transduced but are melanopsin-negative. However, anterograde tracing using rAAV-GFP primarily indicates inputs to sites that register irradiance, including the SCN, vSPZ, VLPO, OPT, 

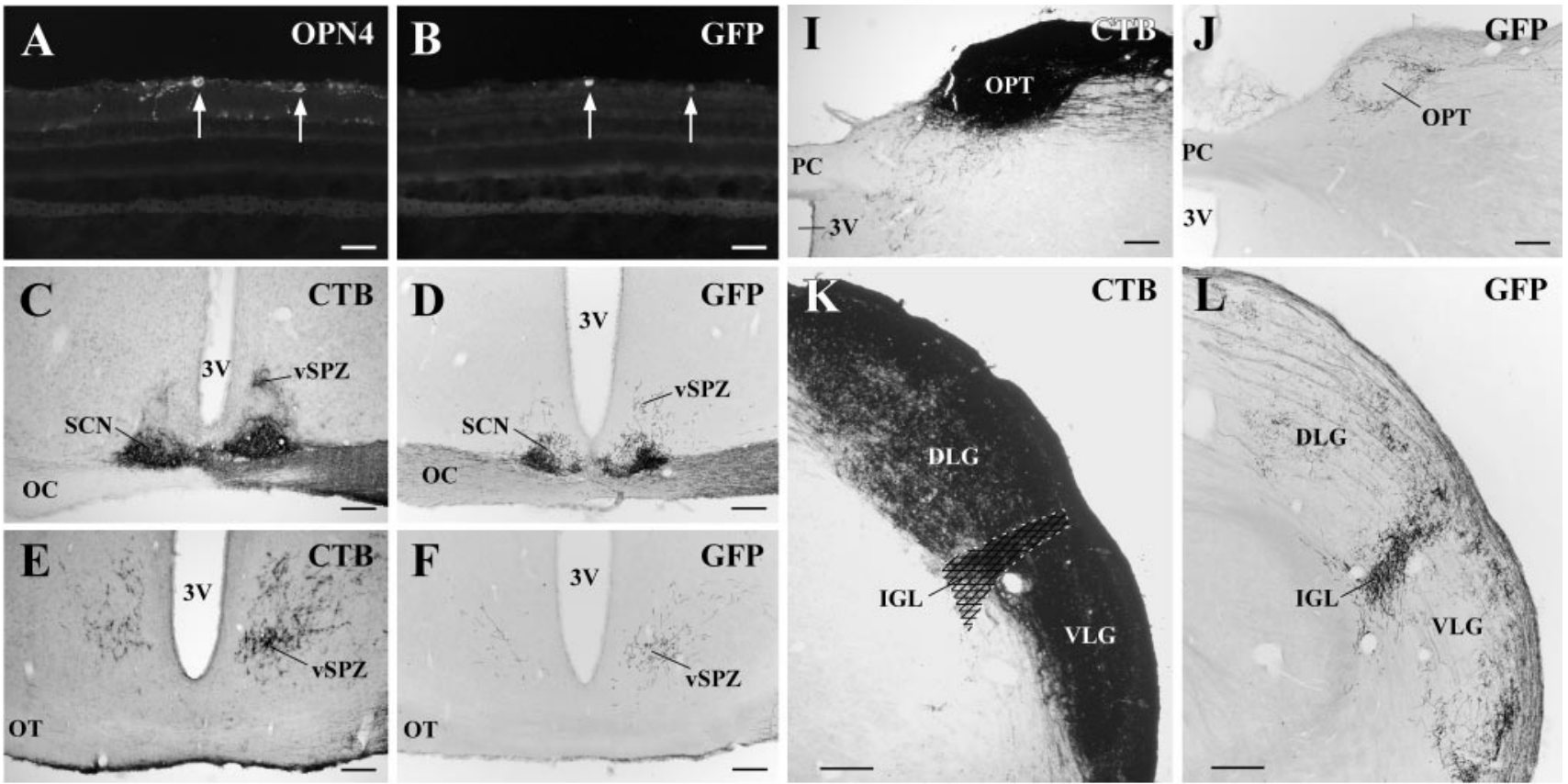

GFP
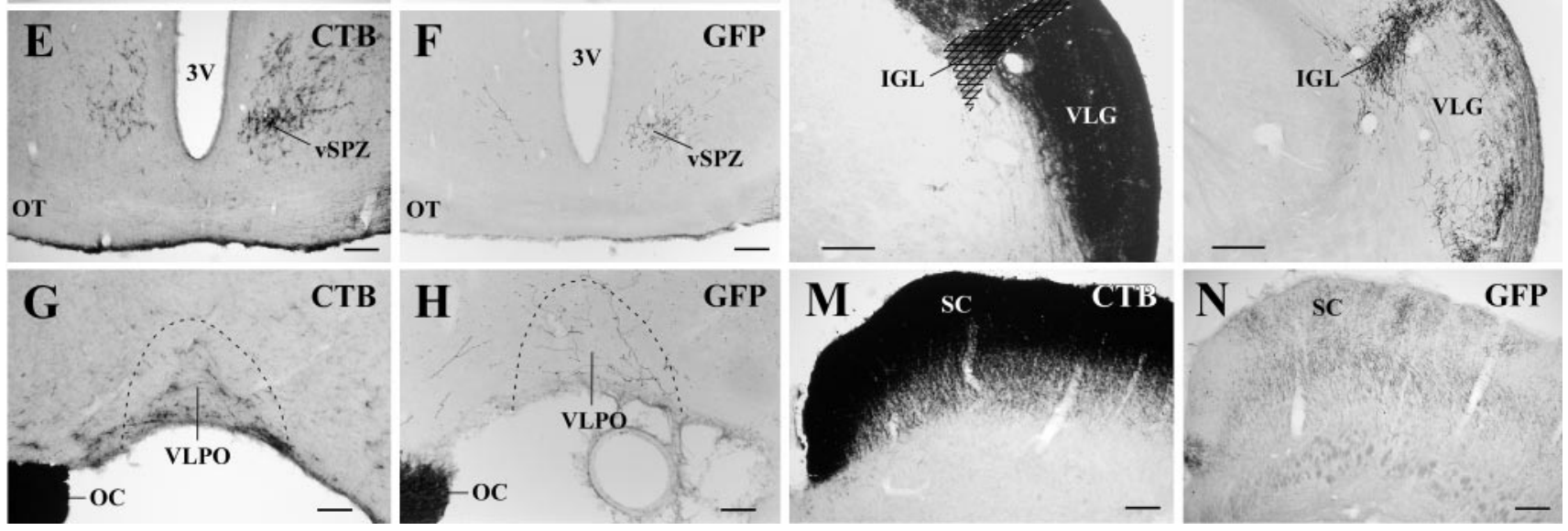

Figure 1. Anterograde tracing from RGGs transduced by rAAV-GFP demonstrates primarily input from melanopsin-expressing RGCS. $A, B, D, F, H, J, L, N$, Case 2472 after intravitreal injection with rAAV-GFP. C, E, G, I, K, M, Case 1172 after intravitreal injection with CTB. After injection of rAAV-GFP into the vitreous body of the right eye, $81 \%$ of GFP-producing cells in the ganglion cell layer ( $B$ ) also contained melanopsin $(A)$. Arrows indicate double-labeled cells. GFP-immunoreactive axonal terminals were observed in the SCN (D), vSPZ (F), VLPO (H), OPT ( J), and IGL (L). In contrast, GFP-labeled projections to the DLG and VLG nuclei were much less intense than those to the IGL. The bilateral SCN and VSPZ and the contralateral VLP0, OPT, IGL, and SC are shown. 3V, Third ventricle; OC, optic chiasm; OPN4, melanopsin; $0 \mathrm{~T}$, optic tract; $\mathrm{PC}$, posterior commissure. Scale bars: $A, B, 50 \mu \mathrm{m} ;(-F, I-N, 200 \mu \mathrm{m} ; G-H, 100 \mu \mathrm{m}$.

and IGL, the expected terminal fields of melanopsin-positive RGCs.

\section{Most retinal ganglion cells that project to the SCN, vSPZ, or VLPO express melanopsin}

To determine whether melanopsin is expressed in subpopulations of RGCs that project to various retinorecipient areas of the brain, FG and/or CTB were injected into the brain to label RGCs retrogradely. Immunocytochemistry was performed for the retrograde tracers, followed by in situ hybridization for melanopsin mRNA. The projections of melanopsin mRNA-containing RGCs were also characterized by performing cell counts, and these data are summarized in Table 1 .

\section{SCN}

The SCN is located in the anterior hypothalamus, just lateral to the third ventricle and immediately dorsal to the optic chiasm. In more caudal sections, the retinorecipient ventrolateral SCN extends dorsally and separates from the optic chiasm. Although the ventrolateral $\mathrm{SCN}$ is most heavily innervated by RGC fibers, RGCs also project to the dorsomedial SCN and vSPZ (see below), which are located just dorsal to the retinorecipient core of the ventrolateral SCN. In four animals, FG injections included the SCN and avoided the optic chiasm (Fig. 2A--D). Although the injection sites were centered in the SCN, the full extent of the tracer diffusion radius encroached on the rostral portion of the vSPZ. Therefore, a small percentage of retrogradely labeled RGCs likely reflects retinal projections to the vSPZ. Because the numbers of retinal axons that innervate the SCN far outnumber those that project to the vSPZ (in animals intravitreally injected with CTB or $\mathrm{rAAV}-\mathrm{GFP}$ ), and the projection to the $\mathrm{vSPZ}$ is predominantly contralateral, we can be confident that the observed pattern of retrogradely labeled RGCs, especially on the ipsilateral side, primarily reflects the subset of retinal efferents that innervate the SCN (compare vSPZ results below). In the eye contralateral to the injection site, $68.1 \pm 5.7 \%$ (SEM) of FGimmunoreactive cells (Fig. $2 E$ ) were also positive for melanopsin transcript $[n=4 ; 7.4 \pm 0.86$ double-labeled RGCs per section (DL/S)] (Fig. 2F). Conversely, $73.7 \pm 3 \%$ of RGCs that expressed melanopsin were also retrogradely labeled. Similar results were observed in the eye ipsilateral to the injection site, in which $72 \pm$ $5.8 \%$ of retrogradely labeled RGCs contained melanopsin mRNA and $70.2 \pm 7.1 \%$ of melanopsin mRNA-containing RGCs were FG immunoreactive ( $n=4 ; 8.2 \pm 1 \mathrm{DL} / \mathrm{S})$, suggesting that many individual melanopsin-expressing RGCs project to the $\mathrm{SCN}$ on both sides of the brain.

$\checkmark S P Z$

The SPZ was first defined by Watts et al. (1987) as the area dorsal and caudal to the SCN that contains the majority of SCN efferents (for review, see Watts, 1991). Lu et al. (2001) have further subdivided the SPZ into ventral and dorsal components on the basis of their differential role in regulating the output of circadian rhythms. The SPZ is defined by the dense column of dorsocau- 
Table 1. Percentage colocalization of melanopsin and retrogradely transported tracer in RGCs that project to various retinorecipient brain regions

\begin{tabular}{|c|c|c|c|c|c|}
\hline $\begin{array}{l}\text { Injection site } \\
\text { per animal }\end{array}$ & $\begin{array}{l}\text { Sections } \\
\text { counted }\end{array}$ & $\begin{array}{l}\text { Double-labeled } \\
\text { RGCs (raw count) }\end{array}$ & $\begin{array}{l}\text { Double-labeled } \\
\text { RGCs per section } \\
\text { (corrected) }\end{array}$ & $\begin{array}{l}\text { Percentage of } \\
\text { tracer-positive RGCs } \\
\text { containing Opn4 mRNA }\end{array}$ & $\begin{array}{l}\text { Percentage of Opn4 } \\
\text { mRNA-positive RGCs } \\
\text { containing tracer }\end{array}$ \\
\hline \multicolumn{6}{|l|}{ SCN contra } \\
\hline 2346 & 5 & 56 & 6.9 & 77.8 & 67.5 \\
\hline 2348 & 5 & 48 & 5.9 & 77.4 & 69.6 \\
\hline 2350 & 5 & 56 & 6.9 & 54.9 & 80.0 \\
\hline 2353 & 5 & 80 & 9.9 & 62.5 & 77.7 \\
\hline Mean & & & $7.4 \pm 0.86$ & $68.1 \pm 5.7$ & $73.7 \pm 3.0$ \\
\hline \multicolumn{6}{|l|}{ SCN ipsi } \\
\hline 2346 & 5 & 46 & 5.7 & 73.0 & 58.2 \\
\hline 2348 & 5 & 61 & 7.5 & 76.3 & 58.7 \\
\hline 2350 & 5 & 75 & 9.3 & 55.6 & 87.2 \\
\hline 2353 & 5 & 83 & 10.3 & 83.0 & 76.9 \\
\hline Mean & & & $8.2 \pm 1.0$ & $72.0 \pm 5.8$ & $70.2 \pm 7.1$ \\
\hline \multicolumn{6}{|l|}{ vSPZ contra } \\
\hline 2548 & 96 & 27 & 0.18 & 79.4 & 2.8 \\
\hline 2550 & 96 & 8 & 0.05 & 57.1 & 0.82 \\
\hline 2567 & 114 & 29 & 0.17 & 85.3 & 1.5 \\
\hline Mean & & & $0.14 \pm 0.04$ & $73.9 \pm 8.6$ & $1.68 \pm 0.57$ \\
\hline \multicolumn{6}{|l|}{ VLPO contra } \\
\hline 2444 & 151 & 14 & 0.06 & 56.0 & 0.61 \\
\hline 2445 & 176 & 24 & 0.09 & 77.4 & 0.67 \\
\hline 2485 & 161 & 11 & 0.05 & 57.9 & 0.53 \\
\hline Mean & & & $0.07 \pm 0.01$ & $63.8 \pm 6.8$ & $0.60 \pm 0.04$ \\
\hline \multicolumn{6}{|l|}{ PTA contra } \\
\hline 2436 & 5 & 51 & 6.6 & 9.0 & 70.8 \\
\hline 2437 & 5 & 68 & 8.8 & 8.3 & 73.9 \\
\hline 2438 & 5 & 23 & 3.0 & 18.7 & 46.0 \\
\hline 2439 & 5 & 48 & 6.2 & 9.0 & 73.8 \\
\hline Mean & & & $6.2 \pm 1.2$ & $11.3 \pm 2.5$ & $66.1 \pm 6.8$ \\
\hline \multicolumn{6}{|l|}{ LGN ipsi } \\
\hline 2454 & 5 & 19 & 2.5 & 35.2 & 17.8 \\
\hline 2455 & 5 & 11 & 1.4 & 19.6 & 16.4 \\
\hline 2457 & 5 & 18 & 2.3 & 34.0 & 18.8 \\
\hline Mean & & & $2.1 \pm 0.33$ & $29.6 \pm 5.0$ & $17.6 \pm 0.68$ \\
\hline
\end{tabular}

Data for the eye contralateral to the injection site are shown for SCN-, VSPZ-, VLPO-, and PTA-injected animals, and results for the ipsilateral eye are shown for SCN- and LGN (IGL)-injected animals (see Results). The column labeled double-labeled RGCs indicates the raw total of cells that contain both retrograde tracer and melanopsin mRNA, but cell counts in the column labeled double-labeled RGCs per section have been corrected using the Abercrombie method (tissue thickness was $20 \mu \mathrm{m}$ for SCN-injected animals and $25 \mu \mathrm{m}$ for all other animals). Mean soma diameter of retrogradely labeled RGCs (in $\mu \mathrm{m}$ ): SCN, 12.34 $\pm 0.47 ; \mathrm{vSPZ}, 13.1 \pm 0.54 ; \mathrm{VLP0}, 12.7 \pm 0.71 ; \mathrm{PTA}, 13.42 \pm 0.43 ; \mathrm{LGN}$ (IGL), 13.31 \pm 0.62. The column labeled percentage of tracer-positive RGCs containing Opn $4 \mathrm{mRNA}$ indicates the percentage of retrogradely labeled cells that contain melanopsin transcript. Conversely, percentage of Opn $4 \mathrm{mRNA}$-positive RGCs containing tracer indicates the percentage of melanopsin-containing RGCs that are also immunoreactive for the retrograde tracer. The percentage colocalization was determined for each animal, and the mean values for each group of injected animals are reported below each group of injected animals. The SEM is shown for all values.

dally directed fibers that leave the dorsal margin of the SCN (at the level of the vSPZ) and then continue dorsally and caudally into the region ventral to the paraventricular nucleus (dorsal SPZ). As defined by Lu et al. (2001), the vSPZ includes the region of SCN terminals in the medial part of the anterior hypothalamic area for $\sim 1 \mathrm{~mm}$ immediately dorsal and caudal to the SCN. Previous studies have described retinal projections to the periSCN and/or SPZ in rats (Johnson et al., 1988; Levine et al., 1991), but immunohistochemical markers have not been used to establish firmly whether the newly defined vSPZ receives direct retinal input. Because VIP-containing neurons, the cell bodies of which are predominantly found in the ventrolateral SCN, project heavily to the SPZ, the vSPZ can be demonstrated by a column of VIP-immunoreactive axons leaving the dorsal margin of the SCN. To examine the projection of RGCs to the vSPZ, we combined immunocytochemistry for anterogradely transported CTB from the retina with immunohistochemical staining for VIP. RGC terminals overlapped extensively with the field of VIPimmunoreactive axons in the contralateral vSPZ but only lightly in the ipsilateral vSPZ (Fig. 3A). This overlap was strongest in the ventral part of the $v S P Z$, in which VIP-immunoreactive terminals leave the dorsal margin of the SCN. We do not believe that this represents a retinal projection to SCN cells that are displaced dorsally into the $\mathrm{vSPZ}$, because the retinal projection remains quite substantial far beyond the borders of the SCN (Fig. 3A). In addition, the SCN is uniquely resistant to injury by injections of ibotenic acid, which destroys all neuronal cell bodies in the vSPZ (Lu et al., 2001). However, without performing electron microscopy, we cannot exclude the possibility that retinal terminals in the vSPZ contact VIP-immunoreactive axons of SCN efferents. Nonetheless, the retinal projection to the vSPZ is well positioned to modify output from the circadian pacemaker.

Having shown that a subset of RGCs projects to the vSPZ, we examined whether these neurons also contain melanopsin mRNA. In three animals, CTB was injected into the vSPZ just dorsal to the caudal retinorecipient region of the SCN (Fig. 3B). Because the densest projection from the SCN terminates in the SPZ, retrogradely labeled cells were observed in the SCN just ventral to the injection site (Fig. 3C). In two animals (2548 and 2550), the injections completely excluded the SCN, and in one other animal, the ventral extent of the injection site encroached on the dorsal border of the SCN (2567). However, the number and pattern of retrogradely labeled RGCs that resulted from injection 2567 were essentially identical to the former injections 

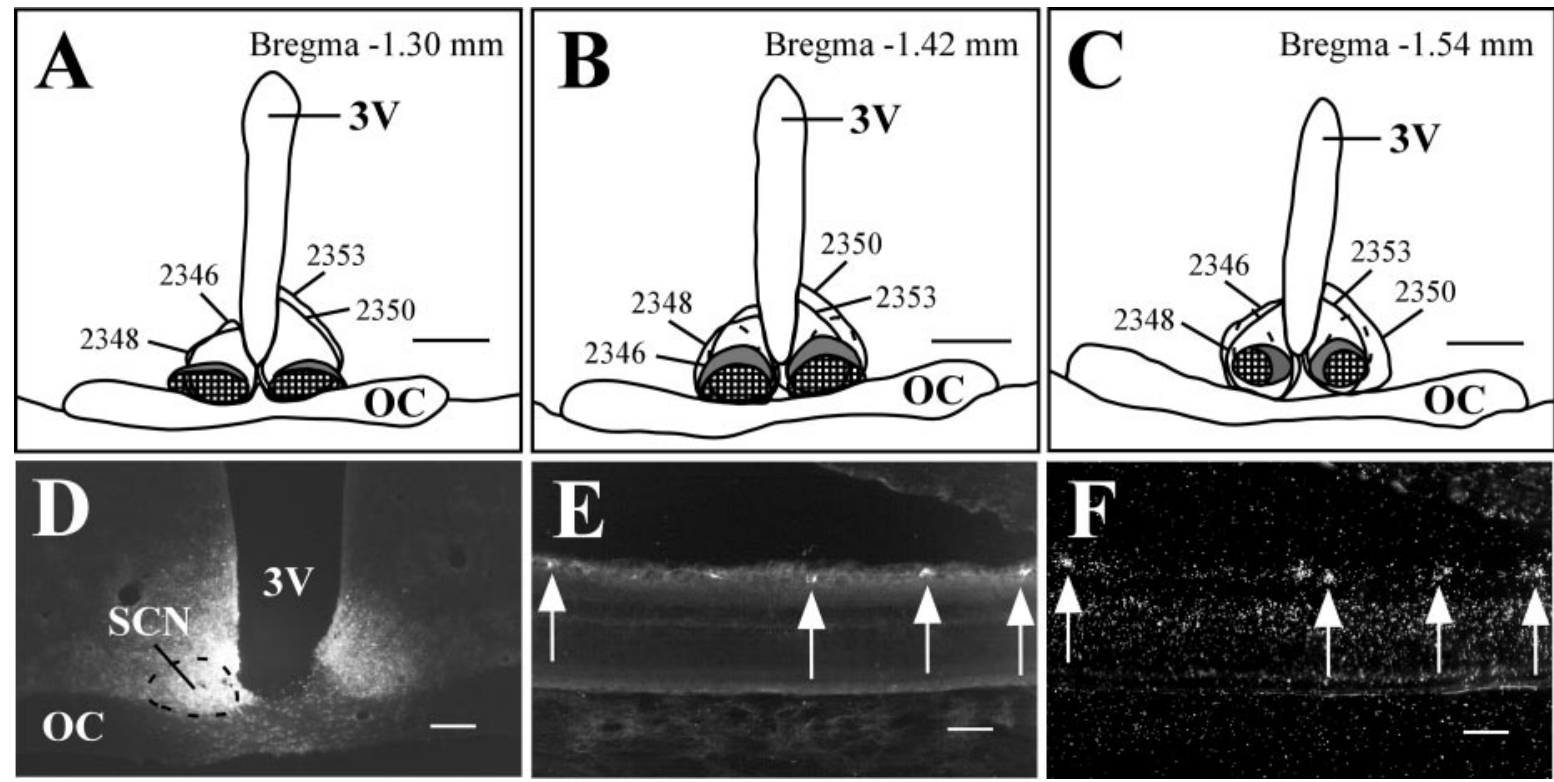

Figure 2. Melanopsin is expressed in the majority of RGCs that project to the SCN. A-C, Camera lucida drawings of coronal brain sections from FG-injected animals. The ventrolateral SCN is checkered, the dorsomedial SCN is colored gray, and the dashed outline dorsal to the SCN indicates the part of the vSPZ that receives relatively sparse retinal input. Smoothly drawn lines indicate injection sites. All injections were made in the right SCN, but some are transposed to the left side for clarity. $D-F$, Case 2348. Injections of FG in the SCN (D) resulted in retrogradely labeled RGCs in the contralateral eye $(E)$ that were also positive for melanopsin transcript $(F)$. Arrows indicate double-labeled cells. $3 \mathrm{~V}$, Third ventricle; $0 C$, optic chiasm. Scale bars: $A-C, 500 \mu \mathrm{m} ; D, 200 \mu \mathrm{m} ; E, F$, $50 \mu \mathrm{m}$.

and not SCN injections (Table 1) and were therefore included in the analysis. Similar to most other retinal projections (and distinct from the SCN), the majority of RGCs that project to the $\mathrm{vSPZ}$ were found to originate in the contralateral eye. In animals 2548,2550 , and $2567,71.3,75.9$, and $93.5 \%$ of retrogradely labeled RGCs originated in the eye contralateral to the injection (mean, $80.2 \pm 6.8 \%$ ). This contrasts markedly with our SCN injections, in which $49.2 \pm 3.4 \%$ of all retrogradely labeled RGCs were located in the contralateral eye. Consistent with these findings and previous reports by Levine et al. (1991), anterograde tracing of retinal efferents using CTB or rAAV-GFP revealed a predominant contralateral projection to the peri-SCN including the vSPZ (Fig. $1 E, F$ ) but approximately equal and bilateral input to the SCN (Fig. 1C,D). To further verify that our SPZ injections did not include the SCN, we examined retrogradely labeled neurons in the dorsal and median raphe nuclei. Previous studies indicate that the lateral cell groups of the dorsal raphe project differentially to the peri-SCN, including the vSPZ, but send relatively fewer axons to the SCN (Moga and Moore, 1997). In contrast, the median raphe projects primarily to the retinorecipient ventrolateral SCN but sends smaller numbers of axons to the peri-SCN. In our SPZ-injected animals, $86.4 \%$ of retrogradely labeled neurons in the dorsal raphe nucleus were found in the lateral cell groups, and the mean number of CTB-immunoreactive cells per section in the dorsal raphe (range, $0-10$ labeled cells per section) was 4.7 times greater than in the median raphe (range, $0-3)$. In addition, the mean number of retrogradely labeled neurons per section was 6.1 times greater in the median raphe of SCN-injected animals (range, 0-15) compared with vSPZinjected animals, indicating a pattern of afferent input consistent with the vSPZ but not the SCN. Similarly, in the IGL ipsilateral to the injection site, the mean number of retrogradely labeled neurons per section was 5.6 times greater in SCN-injected animals (range, 13-49 per section) compared with vSPZ-injected animals (range, 1-16 per section). These data are consistent with anterograde tracing from the IGL to the SCN and peri-SCN, demon- strating that the ventrolateral subdivision of the SCN is most heavily innervated by IGL efferents, and regions dorsal and lateral to the SCN receive considerably fewer afferents from the IGL (Watts, 1991; Moga and Moore, 1997; Moore et al., 2000). In animals that received CTB injections into the vSPZ, $73.9 \pm 8.6 \%$ of retrogradely labeled RGCs (Fig. 3D) also contained melanopsin mRNA $(n=4 ; 0.14 \pm 0.04 \mathrm{DL} / \mathrm{S})$ (Fig. $3 E)$. Conversely, $1.68 \pm 0.57 \%$ of RGCs that contained melanopsin transcript also contained retrogradely transported tracer.

\section{$V L P O$}

The VLPO cluster in the preoptic area is located just lateral to the optic chiasm and rostral to the bulk of the SCN. The densest projection from the retina to the VLPO terminates in the VLPO cluster (Fig. 1G), but RGC varicosities are also present in the extended VLPO, located dorsomedial to the VLPO cluster (Lu et al., 1999, 2002). These findings were replicated in our rAAV-GFP experiments (Fig. $1 \mathrm{H}$ ), suggesting that most of the VLPO projection comes from melanopsin-positive RGCs. Three animals received injections of FG that included both the VLPO cluster and extended VLPO and avoided the optic chiasm (Fig. 4A--D). Consistent with previous findings, a sparse cohort of predominantly contralateral RGCs (mean, $80.1 \pm 4.3 \%$ of all retrogradely labeled RGCs originated in the contralateral eye) was retrogradely labeled from the VLPO (Lu et al., 1999), in which $63.8 \pm 6.8 \%$ of retrogradely labeled RGCs (Fig. $4 E$ ) also expressed melanopsin $(n=3 ; 0.07 \pm 0.01 \mathrm{DL} / \mathrm{S})$ (Fig. $4 F)$. Conversely, $0.60 \pm 0.04 \%$ of melanopsin mRNA-containing RGCs were also positive for retrograde tracer.

\section{A subpopulation of retinal ganglion cells that innervate the PTA or LGN express melanopsin}

\section{PTA}

The PTA includes the medial, anterior, and posterior pretectal nuclei, as well as the OPT, the nucleus of the posterior commis- 


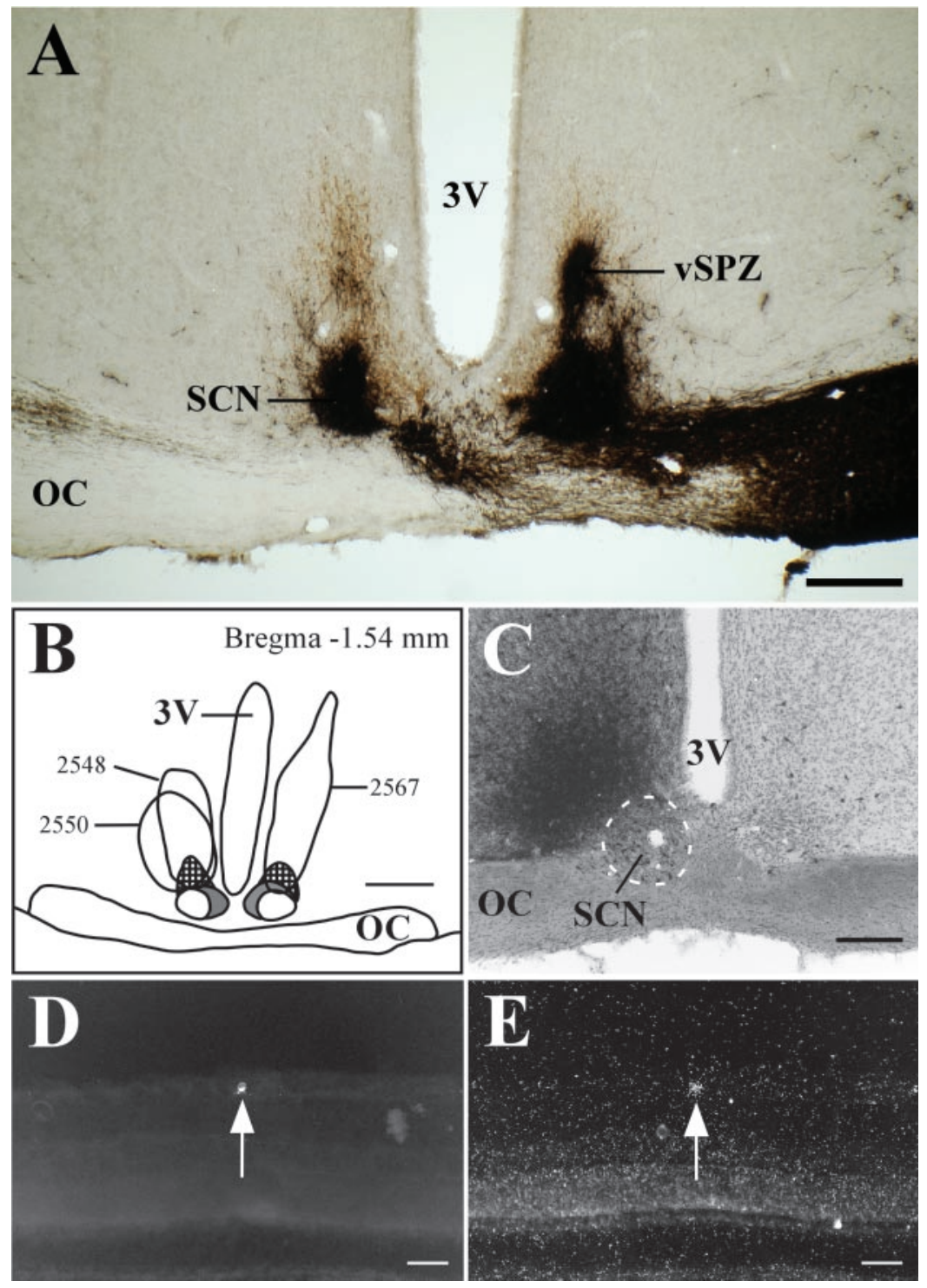

Figure 3. Melanopsin is expressed in the majority of RGCS that project to the vSPZ. A, Retinal terminals (stained in black) overlap extensively with the contralateral vSPZ, as defined by the column of VIP-immuoreactive fibers (stained in brown) leaving the dorsal margin of the SCN. B, Camera lucida drawing of a coronal brain section from BDA-CTB-injected animals. The checkered region indicates the retinorecipient $\mathrm{VSPZ}$, and the gray and white regions ventral to the $\mathrm{V} S P Z$ represent the dorsomedial and ventrolateral SCN, respectively. Smoothly drawn lines indicate injection sites. All injections were made in the right vSPZ, but some are transposed to the left side for clarity. $C-E$, Case 2550. Injections of BDA-CTB in the area including the vSPZ resulted in retrogradely labeled neurons throughout the ipsilateral SCN (C). Retrogradely labeled RGCS in the contralateral eye (D) were positive for melanopsin transcript $(E)$. Arrows indicate a double-labeled cell. 3V, Third ventricle; $0 C$, optic chiasm. Scale bars: $A, C$, $200 \mu \mathrm{m} ; B, 500 \mu \mathrm{m} ; D, E, 50 \mu \mathrm{m}$.

sure, and the nucleus of the optic tract. In the rostral-caudal axis, the pretectal nuclei extend caudally from the rostral-most level of the posterior commissure to the rostral edge of the superior colliculus and mediolaterally from the pineal recess to the lateral posterior thalamic nucleus, posterior limitans thalamic nucleus, and medial geniculate nucleus. The OPT, which is thought to mediate pupillary light responses, is an approximately cigarshaped structure that borders several other pretectal nuclei, which are also densely innervated by retinal efferents (determined by intravitreal injection of CTB) (Figs. $1 \mathrm{I}, 5 \mathrm{~A}-\mathrm{-}$ ) ). However, after intravitreal injection of rAAV-GFP, anterogradely labeled terminals selectively outlined the OPT (Fig. $1 \mathrm{~J}$ ). In four animals, injections of FG were made into the PTA that included the OPT (Fig. 5A--D). The distribution of retrogradely labeled
RGCs was consistent with previous findings, with $\sim 95 \%$ of all retrogradely labeled RGCs in the contralateral eye (Young and Lund, 1998). RGCs projecting to the PTA were unevenly distributed across the retina, such that the majority of retrogradely labeled cells were loosely clustered within one hemisphere of the globe. This is consistent with previous reports indicating that the majority of RGCs projecting to the PTA are located in the inferior and nasal quadrants, and few RGCs are located in the dorsal hemiretina (Young and Lund, 1998). In the eye contralateral to the injection site, $11.3 \pm 2.5 \%$ of retrogradely labeled RGCs (Fig. 5E) were also positive for melanopsin transcript $(n=4 ; 6.2 \pm 1.2$ DL/S) (Fig. 5F). Conversely, $66.1 \pm 6.8 \%$ of melanopsin mRNA-positive RGCs were FG-immunoreactive.

Because the brachium of the SC contains fibers that innervate the SC, and the brachium is located just dorsal to the pretectal nuclei, it was necessary to exclude the possibility that melanopsin mRNA-positive, FGimmunoreactive RGCs project to the SC. Three animals received injections of FG into the SC. As expected, the distribution of retrogradely labeled RGCs differed from that observed for PTA-injected animals. The majority of FG-immunoreactive RGCs were densely clustered in a subsection of the ganglion cell layer, reflecting the retinotopic organization of SC afferents from the retina. Although the density of retrogradely labeled RGCs made it difficult to exclude in all cases that specific retrogradely labeled RGCs were overlaid by some silver grains from an overlying melanopsin mRNA-positive cell, we were unable to find any cases in which a cell body outlined by melanopsin mRNA silver grains was clearly matched by a retrogradely labeled cell body. Similarly, in sections that were immunohistochemically stained for melanopsin, melanopsin-immunoreactive RGCs did not contain retrogradely transported FG. After rAAV-GFP injection into the eye, we found relatively few GFP-positive axons in the SC (Fig. 1N). It is therefore unlikely that FG labeling of melanopsin-positive RGCs observed in animals that received pretectal injections was attributable to involvement of the few axons from melanopsin-containing RGCs passing through to the SC.

\section{$L G N$}

The LGN of the thalamus consists of three major subdivisions that receive input from RGCs. The DLG and VLG subdivisions are large areas that are separated by a thin cellular layer, the IGL. After injections of rAAV-GFP into the eye, GFP-immunoreactive terminals densely innervated the IGL but were sparse in the DLG or VLG, indicating that melanopsin-containing RGCs primarily project to the IGL subdivision of the LGN (Fig. $1 \mathrm{~L}$ ). Five animals received a large injection of FG into the center of the DLG. In two 

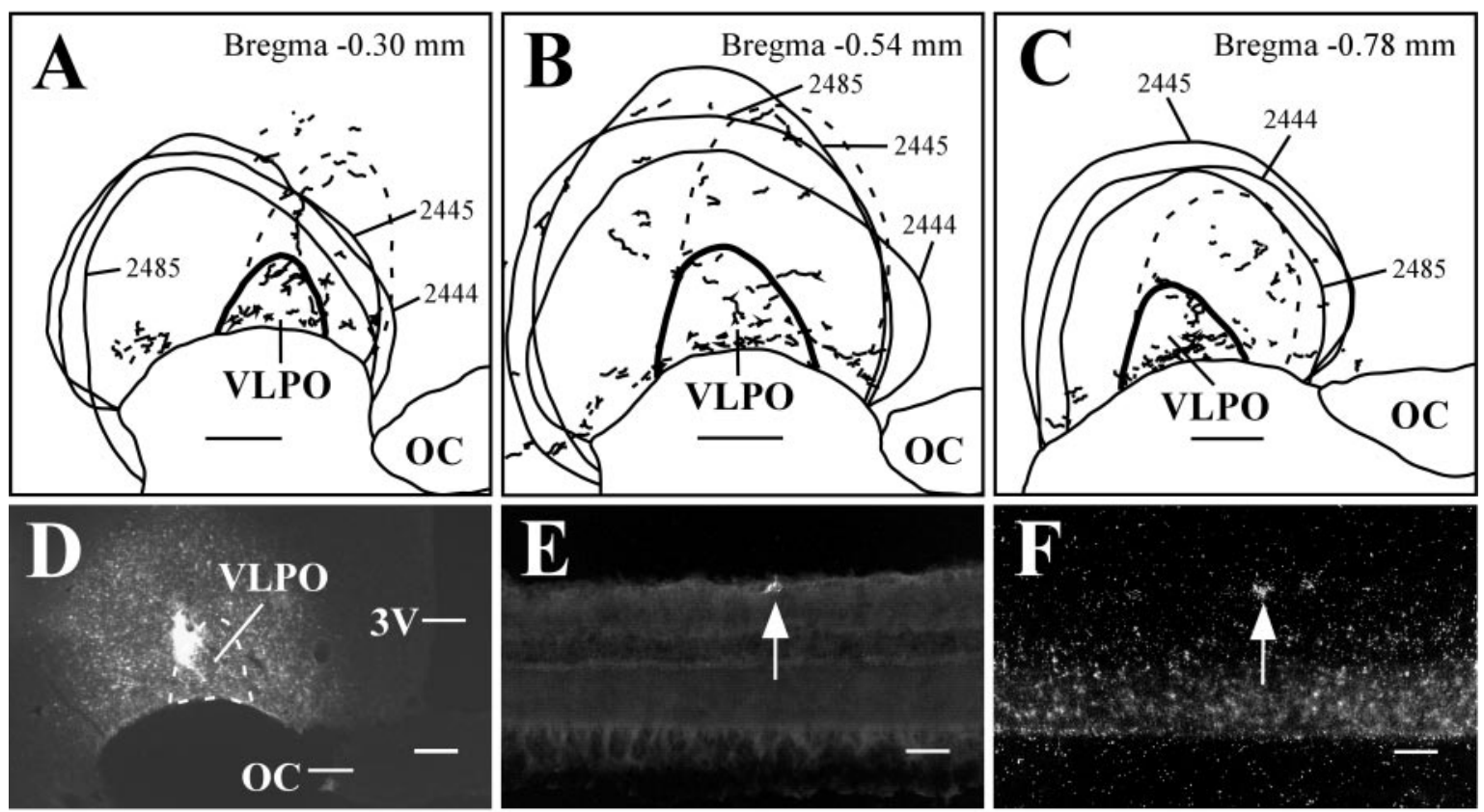

Figure 4. Melanopsin is expressed in the majority of RGCs that project to the VLPO.A-C, Camera lucida drawings of retinal varicosities that terminate in the VLPO region. The VLPO cluster is thickly outlined, the extended VLPO is outlined with the dashed line, and injection sites are indicated by smoothly drawn lines. $D-F$, Case 2445 . Injections of FG in the VLPO region ( $D$ ) resulted in retrogradely labeled RGCs in the contralateral eye ( $E$ ) that were also positive for melanopsin transcript ( $F$. Arrows indicate a double-labeled cell. $3 \mathrm{~V}$, Third ventricle; $0 C, 0$ optic chiasm. Scale bars: $A-D, 200 \mu$ m; $E, F, 50 \mu \mathrm{m}$.
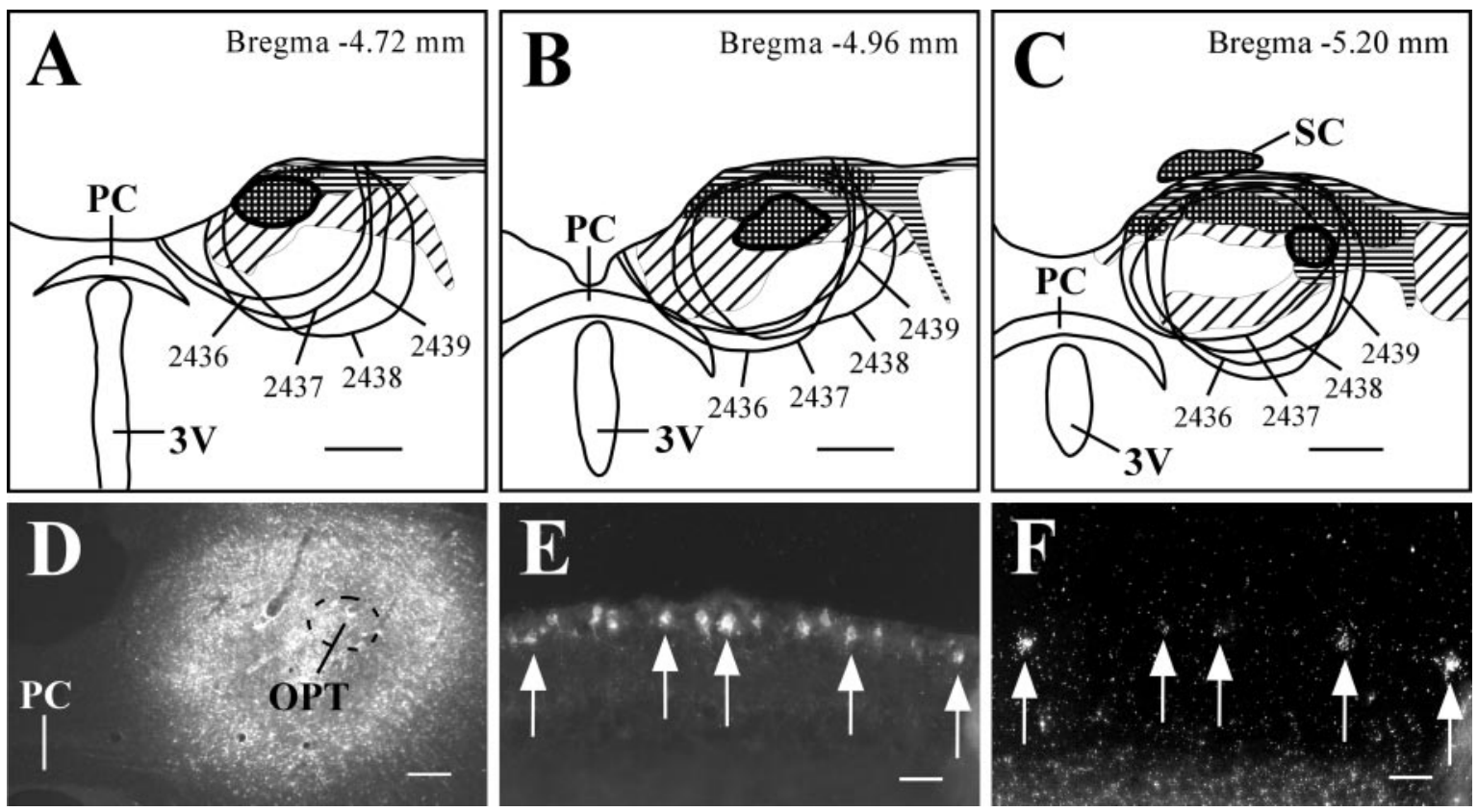

Figure 5. Melanopsin is expressed in a subpopulation of RGCs that project to the PTA. A-C, Camera lucida drawings of coronal brain sections from FG-injected animals. The OPT is thickly outlined, and smoothly drawn lines indicate injection sites. Checkered, horizontal, and diagonal hatching indicate brain regions that are heavily, moderately, or lightly innervated by retinal efferents. $D-F$, Case 2438. Injections of FG in the PTA that included the OPT $(D)$ resulted in retrogradely labeled RGCs in the contralateral eye $(E)$ that were also positive for melanopsin transcript $(F)$. Arrows indicate double-labeled cells. $3 V$, Third ventricle; PC, posterior commissure. Scale bars: $A--C, 500 \mu \mathrm{m} ; D, 200 \mu \mathrm{m} ; E, F, 50 \mu \mathrm{m}$.

animals, injections were confined to the DLG and did not retrogradely label neurons in the contralateral IGL; in three animals the injection site included the IGL and the dorsal edge of the VLG, resulting in retrogradely labeled cells in the contralateral IGL (Fig. 6A,B). Because nearly all DLG and VLG afferents from the retina are contralateral (>95\%), and RGCs from each eye project bilaterally and equally to the rostral and midportion level of each
IGL, we examined retrogradely labeled RGCs in the ipsilateral eye, which likely primarily reflect RGCs that project to the IGL. However, we cannot exclude the possibility that a small minority of retrogradely labeled RGCs project to the DLG or the dorsal edge of the VLG. In the eye ipsilateral to the injection site, FGimmunoreactive RGCs were typically localized to a small subsection of the retina, and injections that were confined to the DLG 


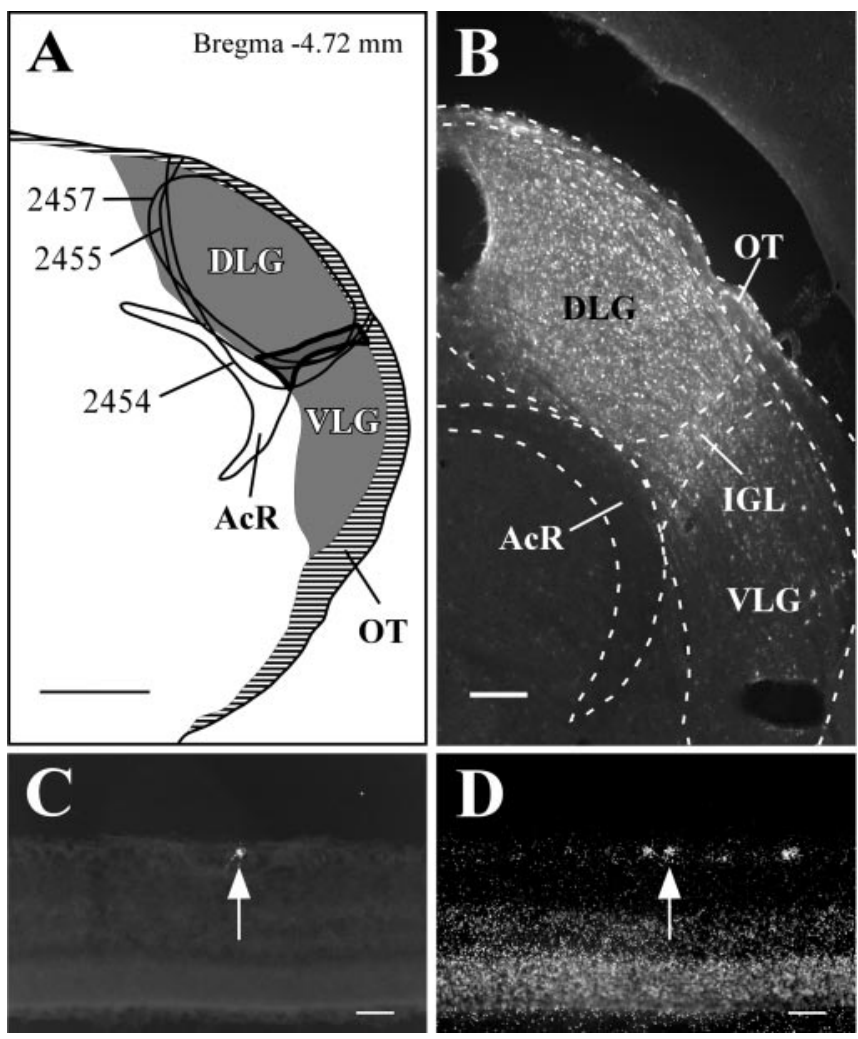

Figure 6. Melanopsin is expressed in a subpopulation of RGCS that project to the LGN. $A_{\text {, }}$ Camera lucida drawing of a coronal brain section from FG-injected animals. The IGL is thickly outlined, and smoothly drawn lines indicate injection sites. Gray regions indicate brain regions that receive heavy input from the retina, and horizontal lines indicate the optic tract. $B-D$, Case 2455. Injections of FG in the LGN that included the IGL $(B)$ resulted in retrogradely labeled RGCS in the ipsilateral eye $(C)$ that were also positive for melanopsin transcript $(D)$. Arrows indicate a double-labeled cell. AcR, Acoustic radiation; 0T, optic tract. Scale bars: $A, 500 \mu \mathrm{m} ; B, 200 \mu \mathrm{m}$; $C, D, 50 \mu \mathrm{m}$.

$(n=2)$ did not retrogradely label RGCs that expressed melanop$\sin$. However, for injections that included the IGL (Fig. 6A,B), $29.6 \pm 5 \%$ of retrogradely labeled RGCs (Fig. 6C) also expressed melanopsin $(n=3 ; 2.1 \pm 0.33 \mathrm{DL} / \mathrm{S})$ (Fig. $6 \mathrm{D})$. Conversely, $17.6 \pm 0.68 \%$ of RGCs that contained melanopsin transcript were also FG immunoreactive. Thus, the RGCs that project to the DLG subdivision of the LGN do not contain melanopsin, but a substantial proportion of RGCs that do contain melanopsin innervate the IGL.

\section{A subpopulation of retinal ganglion cells that express melanopsin projects to both the SCN and PTA}

Having shown that the majority of RGCs that express melanopsin project to the SCN, and the majority of melanopsin mRNAcontaining RGCs also innervate the PTA, we examined to what extent these two projections arise as branched collaterals from the same population of RGCs. FG and CTB were injected into the ipsilateral SCN (Fig. 7A--C,G) and PTA (Fig. 7D-- F, H), respectively, to retrogradely label the corresponding subpopulations of RGCs that project to these brain regions. In case 2512, the FG injection was completely confined to the SCN and peri-SCN region (including part of the vSPZ) and avoided the optic chiasm. In the eye contralateral to the injections in case 2512, we found that $28.6 \%$ of retrogradely labeled RGCs from the SCN were also CTB immunoreactive, demonstrating that a subpopulation of RGCs sends axons that bifurcate and project to both the SCN and
OPT $(5.3 \pm 1.8 \mathrm{DL} / \mathrm{S})$. Conversely, $28.9 \%$ of retrogradely labeled RGCs from the PTA were immunoreactive for retrogradely transported FG from the SCN. Having shown that a subset of RGCs sends bifurcating axons to the SCN and PTA, we examined these double-labeled cells for melanopsin transcript. In case 2512, we observed that $37.5 \%$ of FG- and CTB-immunoreactive RGCs contained melanopsin transcript, and $16.1 \%$ of RGCs that contained melanopsin mRNA were positive for both neuronal tracers (1.2 triple-labeled RGCs per section).

In animals 2527, 2535, and 2537, injections were primarily confined to the SCN and peri-SCN, but the ventral-most extent of the tracer diffusion radius did encroach on the dorsal border of the optic chiasm (Fig. 7A--C). Because the number of FGimmunoreactive RGCs was larger in these cases (indicating some uptake by the optic nerve fibers in the optic chiasm), the percentage of FG-positive RGCs that also contained CTB (13.4 $\pm 1.4 \%)$ was smaller than in case 2512 (28.6\%), in which the optic chiasm was not involved, suggesting that approximately half of the FGlabeled RGCs in these cases were attributable to uptake in the optic chiasm. As a consequence, the percentage of doubly retrogradely labeled cells that contained melanopsin mRNA was nearly twice $(62.5 \pm 10.7 \%)$ that in case $2512(37.5 \%)$, and the percentage of melanopsin-positive RGCs that contained both tracers was also $\sim 60 \%$ higher $(26.2 \pm 7.9 \%)$ than in case 2512 (16.1\%). Allowing for this excess labeling, these results confirm the observations in case 2512 that $\sim 40 \%$ of the RGCs that project to both the SCN and OPT also express melanopsin mRNA, and $\sim 20 \%$ of the RGCs that contain melanopsin project to both sites.

\section{Discussion}

In this study, we have explored the range of projections from melanopsin-containing RGCs to different retinorecipient brain targets. Our findings demonstrate that melanopsin mRNA-containing RGCs project extensively to sites that register irradiance, including the SCN, vSPZ, VLPO, PTA, and the IGL subdivision of the LGN. However, sites that participate primarily in pattern vision, such as the DLG and SC, receive few inputs from the population of RGCs that express melanopsin. In fact, the majority of melanopsincontaining RGCs project to each of the SCN and contralateral PTA, and nearly $20 \%$ project to the ipsilateral IGL. This observation suggests that individual melanopsin-containing RGCs send axon collaterals to multiple targets, and we found that $20 \%$ of RGCs that express melanopsin project to both the SCN and PTA. Similarly, previous studies showed that the SCN and IGL share inputs from single RGCs (Pickard, 1985). We propose that melanopsincontaining RGCs and their brain targets constitute a retinal irradiance system that functions independently of the pattern vision system and may drive or contribute to a variety of light-induced responses, including photic circadian entrainment, melatonin suppression, negative masking, the pupillary light reflex, and the regulation of sleep-wake states.

\section{Technical considerations}

A potential pitfall of injecting retrograde tracer into a small brain region is that the tracer diffuses locally to adjacent structures. This problem was of particular concern because most of our injection targets border other retinorecipient areas. For example, the SCN and vSPZ border each other, the SCN and VLPO border the optic chiasm, the OPT borders other pretectal nuclei and the brachium of the SC, and the IGL borders the DLG and VLG. In particular, the size and shape of the OPT and IGL are such that the injected tracer invariably labels part of the nearby retinorecipient brain areas. Therefore, the population of retrogradely labeled RGCs includes ef- 

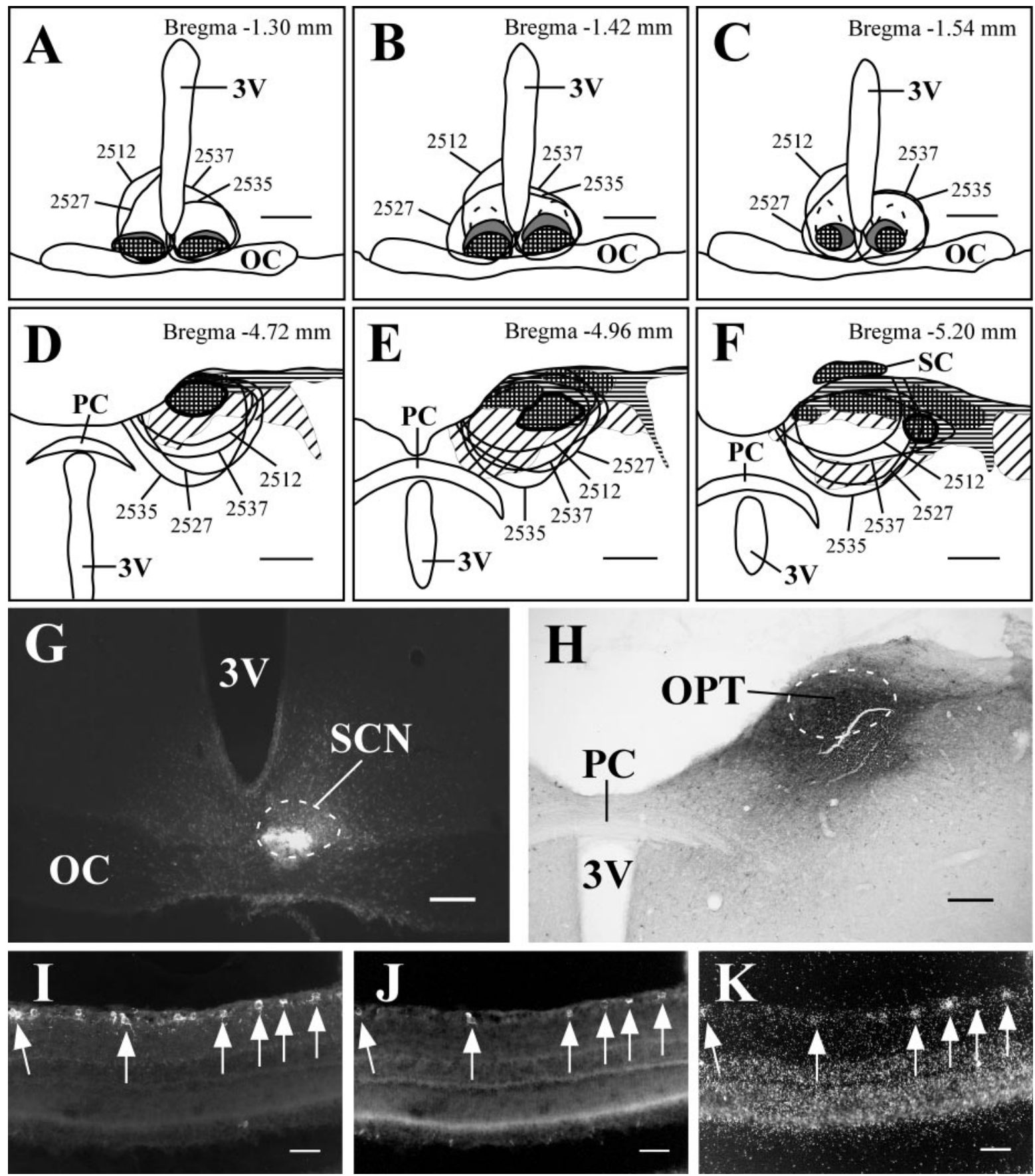

Figure 7. Melanopsin is expressed in a subset of RGCS, the axons of which bifurcate and project to both the SCN and PTA. A-F, Camera lucida drawings of coronal sections from FG- and CTB-injected animals. The retinorecipient ventrolateraI SCN $(A-C)$ is checkered, the dorsomedial SCN is colored gray, and the outlined region dorsal to the SCN indicates the sparsely innervated vSPZ. Smoothly drawn lines indicate FG injection sites. All injections were made in the left SCN, but some are transposed to the right side for clarity. The OPT is thickly outlined ( $D-F$ ), and smoothly drawn lines indicate CTB injection sites. Checkered, horizontal, and diagonal hatching indicate brain regions that are heavily, moderately, or lightly innervated by retinal efferents. $G-K$, Case 2537. Injections of $\mathrm{FG}$ and $\mathrm{CTB}$ in the SCN $(G)$ and PTA $(H)$, respectively, resulted in retrogradely labeled RGCs in the contralateral eye that were both FG-immunoreactive $(I)$ and $C T B$-immunoreactive $(J)$ and were positive for melanopsin transcript ( $K$ ). Arrows indicate triple-labeled cells. 3V, Third ventricle; $0 C$, optic chiasm; PC, posterior commissure. Scale bars: $A-F, 500 \mu \mathrm{m} ; \mathrm{G}-H, 200 \mu \mathrm{m}$; $I-K, 50 \mu \mathrm{m}$.

ferents that project to the target injection site as well as some RGCs that innervate adjacent structures. We found that injections that included the OPT and IGL resulted in retrogradely labeled RGCs that contained melanopsin transcript, but control injections into the DLG and SC did not result in double-labeled RGCs. Hence, the double-labeled neurons clearly project to the PTA and IGL, but we cannot be sure what percentage of the singly retrogradely labeled RGCs target these structures.
Although the percentage of colocalization of the retrograde tracer and melanopsin mRNA was consistent and reproducible using our methods, our calculations likely underestimate the actual percentage of colocalization resulting from technical factors that limit the efficiency of the combined labels. Performing immunocytochemistry for the retrograde tracer decreased the signal for melanopsin transcript and, therefore, the number of discernable melanopsin mRNA-positive RGCs compared with tissue 
that was used only for in situ hybridization ( $\sim 15 \%$ reduction). However, performing immunocytochemistry for FG allowed us to identify retrogradely labeled cells that otherwise would have gone undetected because of light labeling.

\section{Potential redundancy of phototransduction in irradiance-dependent pathways}

Our data indicate that melanopsin is expressed in RGCs that contribute to a wide range of pathways that mediate irradiancedependent responses. In intact animals, the melanopsincontaining RGCs may be dominated by inputs from rods and cones. For example, in dark- and light-adapted rats, the action spectrum for visually responsive SCN neurons conforms to the sensitivity of rhodopsin and cone opsins, respectively (Aggelopoulos and Meissl, 2000). However, in animals that are deficient in rods and cones, the action spectrum for phase resetting and the pupillary light reflex shifts to shorter wavelengths consistent with the action spectrum for melanopsin-containing RGCs (Yoshimura and Ebihara, 1996; Lucas et al., 2001; Berson et al., 2002). Recent studies in Opn4 null mice show that melanopsin is required for normal light-induced circadian phase shifts and pupillary constriction in response to bright light (Panda et al., 2002; Ruby et al., 2002; Lucas et al., 2003). However, Opn $4^{-/-}$animals still show photic entrainment and a normal pupillary light reflex under low to moderate light, indicating redundancy of phototransduction in these irradiance-dependent responses.

Although melanopsin is a primary candidate for mediating irradiance-dependent responses in animals that lack rods and cones, it is possible that other photopigments may also contribute to this pathway. Cryptochomes 1 and 2 are also expressed in the mammalian inner retina (Miyamoto and Sancar, 1998), and retinal degenerate $(r d / r d)$ mice lacking cryptochromes exhibit reduced pupillary light responses (Van Gelder et al., 2003). However, there is not yet evidence that the cryptochromes form functional photopigments in the mammalian retina, nor is it known whether they are found in RGCs that contribute to the irradiance-dependent pathways.

\section{The role for melanopsin in novel projections to the vSPZ and VLPO}

We have characterized novel projections of melanopsincontaining RGCs to the vSPZ and VLPO in the anterior hypothalamus. Ablation of the retinorecipient vSPZ specifically reduces the circadian rhythms of sleep and locomotor activity, whereas lesions in the dorsal SPZ primarily reduce the core body temperature rhythm ( $\mathrm{Lu}$ et al., 2001). The direct projection of melanopsin-containing RGCs to the vSPZ could therefore modify (or mask) photic circadian entrainment of sleep and locomotor activity rhythms by acting downstream of the circadian oscillator.

A nonclassical photopigment has also been implicated in light-induced suppression of locomotor activity in rodents, termed negative masking. This behavior persists in $r d / r d$, rodless and coneless $(\mathrm{rdta} / \mathrm{cl})$, SCN-lesioned, Cry $1^{-/-} / \mathrm{Cry} 2^{-/-}$, and $O p n 4^{-1-}$ animals, suggesting that negative masking may be mediated by redundant phototransduction pathways (Mrosovsky, 1994, 2001; Mrosovky et al., 1999, 2001; Redlin and Mrosovsky, 1999; Panda et al., 2002). The majority of RGCs that project to the vSPZ express melanopsin and may contribute to negative masking directly via this pathway (Kramer et al., 2001).

Melanopsin transcript is contained in the majority of RGCs that project to the VLPO. The VLPO neurons contain the inhibitory neurotransmitters galanin and GABA and are thought to inhibit the ascending arousal system, thereby producing sleep (Sherin et al., 1996, 1998; Lu et al., 2000, 2002). Few studies have addressed circadian-independent direct effects of light on the regulation of sleep. However, in albino rats that sleep during the light cycle, an acute exposure to light suppresses rapid eye movement sleep (Fishman and Roffwarg, 1972; Benca et al., 1996, 1998), which may account for the tendency of most animals to close their eyes while sleeping. Although the retinal projection to the VLPO is relatively sparse, axon terminals are found among Fos-immunoreactive neurons in sleeping animals, and retinal terminal boutons form appositions with galanin-immunoreactive cell bodies and proximal dendrites (Lu et al., 1999). Thus, this pathway provides a substrate by which melanopsin-containing RGCs may play a direct role in the modulation of sleep-wake states.

\section{The role for melanopsin in projections to the SCN, IGL, and PTA}

Melanopsin is found in the majority of RGCs that project to the SCN, the site of the master circadian pacemaker in mammals (Gooley et al., 2001; Hannibal et al., 2002). These melanopsincontaining RGCs are intrinsically photosensitive and may function as photoreceptors for circadian entrainment (Berson et al., 2002; Hattar et al., 2002). A definitive role for melanopsin in the circadian phototransduction pathway has recently been demonstrated in Opn4 $4^{-1-}$ mice (Panda et al., 2002; Ruby et al., 2002). Light-induced phase shifts are attenuated in Opn4 null animals, indicating that melanopsin is required for normal circadian entrainment. The projection of melanopsin-containing RGCs to the IGL also suggests a role for melanopsin in the regulation of circadian rhythms. Although the IGL itself is not required for photic circadian entrainment, the IGL conveys both photic and nonphotic information to the circadian clock via the geniculohypothalamic tract (for review, see Morin, 1994; Mrosovsky, 1996; Harrington, 1997; Hastings et al., 1997). Thus, in addition to its putative role in circadian phase shifting, the IGL has been implicated in the regulation of circadian period, phase angle, and integration of photoperiodic information.

Melanopsin has recently been shown to contribute to the afferent limb of the pupillary light reflex (Lucas et al., 2003). In Opn4 null mice, the pupillary light reflex is diminished in response to bright light. In rats, the firing rate of neurons in the OPT increases as luminance is increased, and unilateral lesions of the OPT greatly reduce both the direct and consensual pupillary light reflexes (Clarke and Ikeda, 1985; Young and Lund, 1994). The OPT projects to the Edinger-Westphal nucleus, which sends efferents to the ciliary ganglion (Klooster et al., 1995). The parasympathetic postganglionic neurons innervate the iris sphincter muscle, resulting in pupillomotor constriction. The projection of melanopsin-containing RGCs to the OPT is consistent with melanopsin being able to drive the pupillary light reflex in the absence of input from classical photoreceptors (Lucas et al., 1999).

\section{Axonal projections of melanopsin-containing RGCs are highly collateralized}

We have demonstrated that $\geq 65-75 \%$ of RGCs that express melanopsin project to each of the contralateral and ipsilateral SCN as well as to the contralateral PTA, and nearly $20 \%$ project to the ipsilateral IGL. It is therefore likely that most axonal projections of melanopsin-containing RGCs are highly collateralized. Previous studies have shown that a subset of RGCs sends bifurcating axons to the SCN and IGL (Pickard, 1985). We have shown that $\sim 20 \%$ of melanopsin-containing RGCs project to both the 
SCN and PTA, suggesting that these RGCs may drive photic circadian entrainment and the pupillary light reflex simultaneously in animals lacking input from classical photoreceptors. The spectral sensitivity of RGCs that contain melanopsin $\left(\lambda_{\max }=484 \mathrm{~nm}\right)$ is consistent with the range reported for light-induced circadian phase shifts and the pupillary light reflex in wild-type and retinal degenerate rodents, suggesting that melanopsin might contribute to both responses $\left(\lambda_{\max }=480-510\right.$ nm) (Takahashi et al., 1984; Provencio and Foster, 1995; Yoshimura and Ebihara, 1996; Lucas et al., 2001). Similarly, activation of melanopsin-containing RGCs could drive light-induced suppression of melatonin, which is driven by relayed input from the SCN (Czeisler et al., 1995; Lucas et al., 1999; Brainard et al., 2001; Thapan et al., 2001). Our data show that melanopsin-containing RGCs send highly collateralized axonal projections to multiple brain sites that register irradiance, indicating that these RGCs are specialized to transmit nonvisual photic information.

\section{Multiple parallel pathways for photic input to the SCN}

The primary mode of circadian entrainment is likely mediated by the monosynaptic retinohypothalamic projection to the SCN. However, the presence of melanopsincontaining RGCs projecting to other sites could provide multiple parallel pathways for regulation of SCN activity (Fig. 8). In its role as the circadian pacemaker, the SCN must integrate photic signals from RGCs and other retinorecipient areas of the brain. We found melanopsin transcript in RGCs that project to the vSPZ, IGL, and PTA, which all give rise to fibers that innervate the retinorecipient region of the SCN (Moga and Moore, 1997; Moore et al., 2000; Krout et al., 2002). The major output from the SCN is to the SPZ, which receives input from melanopsin-positive RGCs and afferents from the IGL (Moga and Moore, 1997; Moore et al., 2000). The caudal portion of the IGL also gives rise to fibers that terminate in the OPT (Moga and Moore, 1997). The SPZ and SCN both project to the VLPO, which is innervated by melanopsin-positive RGCs (Gaus and Saper, 1998; Novak and Nunez, 2000; Chou et al., 2002). Interestingly, nearly all of the known brain regions that receive direct input from RGCs that express melanopsin also project to other areas innervated by melanopsin-positive RGCs. These data suggest that the projections of the melanopsin-containing RGCs define an irradiance-driven network distinct from the visual system for detection of movement or patterns. The melanopsin-containing RGCs appear to play a crucial role in the regulation of light-driven responses, and these responses are apparently generated both by multiple sources of photic reception in the retina and multiple levels of integration of photic information in the brain.

\section{References}

Abercrombie M (1946) Estimation of nuclear population from microtome sections. Anat Rec 94:239-247.

Aggelopoulos NC, Meissl H (2000) Responses of neurones of the rat suprachiasmatic nucleus to retinal illumination under photopic and scotopic conditions. J Physiol (Lond) 523:211-222.
Ali RR, Reichel MB, De Alwis M, Kanuga N, Kinnon C, Levinsky RJ, Hunt DM, Bhattacharya SS, Thrasher AJ (1998) Adeno-associated virus gene transfer to mouse retina. Hum Gene Ther 9:81-86.

Auricchio A, Kobinger G, Anand V, Hildinger M, O'Connor E, Maguire AM, Wilson JM, Bennett J (2001) Exchange of surface proteins impacts on viral vector cellular specificity and transduction characteristics: the retina as a model. Hum Mol Genet 10:3075-3081.

Benca RM, Overstreet DE, Gilliland MA, Russell D, Bergmann BM, Obermeyer WH (1996) Increased basal REM sleep but no difference in dark induction or light suppression of REM sleep in Flinders rats with cholinergic supersensitivity. Neuropsychopharmacology 15:45-51.

Benca RM, Gilliland MA, Obermeyer WH (1998) Effects of lighting conditions on sleep and wakefulness in albino Lewis and pigmented Brown Norway rats. Sleep 21:451-460.

Berson DM, Dunn FA, Takao M (2002) Phototransduction by retinal ganglion cells that set the circadian clock. Science 295:1070-1073.

Brainard GC, Hanifin JP, Greeson JM, Byrne B, Glickman G, Gerner E, Rollag MD (2001) Action spectrum for melatonin regulation in humans: evidence for a novel circadian photoreceptor. J Neurosci 21:6405-6412.

Chou TC, Bjorkum AA, Gaus SE, Lu J, Scammell TE, Saper CB (2002) Afferents to the ventrolateral preoptic nucleus. J Neurosci 22:977-990.

Clarke RJ, Ikeda H (1985) Luminance and darkness detectors in the olivary and posterior pretectal nuclei and their relationship to the pupillary light reflex in the rat. I. Studies with steady luminance levels. Exp Brain Res 57:224-232.

Czeisler CA, Shanahan TL, Klerman EB, Martens H, Brotman DJ, Emens JS, Klein T, Rizzo III JF (1995) Suppression of melatonin secretion in some blind patients by exposure to bright light. N Engl J Med 332:6-11.

Dreyer EB, Vorwerk CK, Zurakowski D, Simon PD, Bennett J (1999) Infection with adeno-associated virus may protect against excitotoxicity. NeuroReport 10:2887-2890.

Dudus L, Anand V, Acland GM, Chen SJ, Wilson JM, Fisher KJ, Maguire AM, Bennett J (1999) Persistent transgene product in retina, optic nerve and brain after intraocular injection of rAAV. Vision Res 39:2545-2553. 
Elmquist JK, Saper CB (1996) Activation of neurons projecting to the paraventricular hypothalamic nucleus by intravenous lipopolysaccharide. J Comp Neurol 374:315-331.

Fischer D, Heiduschka P, Thanos S (2001) Lens-injury-stimulated axonal regeneration throughout the optic pathway of adult rats. Exp Neurol $172: 257-272$.

Fishman R, Roffwarg HP (1972) REM sleep inhibition by light in the albino rat. Exp Neurol 36:166-178.

Freedman MS, Lucas RJ, Soni B, von Schantz M, Munoz M, David-Gray Z, Foster R (1999) Regulation of mammalian circadian behavior by nonrod, non-cone, ocular photoreceptors. Science 284:502-504.

Gaus SE, Saper CB (1998) Efferent connections from the suprachiasmatic nucleus to the ventrolateral preoptic nucleus in the rat. Soc Neurosci Abstr 24:1920.

Geuna S (2000) Appreciating the difference between design-based and model-based sampling strategies in quantitative morphology of the nervous system. J Comp Neurol 427:333-339.

Gooley JJ, Lu J, Chou TC, Scammell TE, Saper CB (2001) Melanopsin in cells of origin of the retinohypothalamic tract. Nat Neurosci 4:1165.

Grant CA, Ponnazhagan S, Wang XS, Srivastava A, Li T (1997) Evaluation of recombinant adeno-associated virus as a gene transfer vector for the retina. Curr Eye Res 16:949-956.

Guy J, Qi X, Muzyczka N, Hauswirth WW (1999) Reporter expression persists 1 year after adeno-associated virus-mediated gene transfer to the optic nerve. Arch Ophthalmol 117:929-937.

Hankins MW, Lucas RJ (2002) The primary visual pathway in humans is regulated according to long-term light exposure through the action of a nonclassical photopigment. Curr Biol 12:191-198.

Hannibal J, Hindersson P, Knudsen SM, Georg B, Fahrenkrug J (2002) The photopigment melanopsin is exclusively present in pituitary adenylate cyclase-activating polypeptide-containing retinal ganglion cells of the retinohypothalamic tract. J Neurosci 22:RC191.

Harrington ME (1997) The ventral lateral geniculate nucleus and the intergeniculate leaflet: interrelated structures in the visual and circadian systems. Neurosci Biobehav Rev 21:705-727.

Harvey AR, Kamphuis W, Eggers R, Symons NA, Blits B, Niclou S, Boer GJ, Verhaagen J (2002) Intravitreal injection of adeno-associated viral vectors results in the transduction of different types of retinal neurons in neonatal and adult rats; a comparison with lentiviral vectors. Mol Cell Neurosci 21:141-157.

Hastings MH, Duffield GE, Ebling FJ, Kidd A, Maywood ES, Schurov I (1997) Non-photic signaling in the suprachiasmatic nucleus. Biol Cell 89:495-503.

Hattar S, Liao HW, Takao M, Berson DM, Yau KW (2002) Melanopsincontaining retinal ganglion cells: architecture, projections, and intrinsic photosensitivity. Science 295:1065-1070.

Johnson RF, Morin LP, Moore RY (1988) Retinohypothalamic projections in the hamster and rat demonstrated using cholera toxin. Brain Res 462:301-312.

Kitt C (1988) Immunocytochemical visualization of cholinergic fibers in monkey neocortex: enhanced visualization using silver nitrate. Soc Neurosci Abstr 14:631.

Klooster J, Vrensen GF, Muller LJ, van der Want JJ (1995) Efferent projections of the olivary pretectal nucleus in the albino rat subserving the pupillary light reflex and related reflexes. A light microscopic tracing study. Brain Res 688:34-46.

Kramer A, Yang FC, Snodgrass P, Li X, Scammell TE, Davis FC, Weitz CJ (2001) Regulation of daily locomotor activity and sleep by hypothalamic EGF receptor signaling. Science 294:2511-2515.

Krout KE, Kawano J, Mettenleiter TC, Loewy AD (2002) CNS inputs to the suprachiasmatic nucleus of the rat. Neuroscience 110:73-92.

Levine JD, Weiss ML, Rosenwasser AM, Miselis RR (1991) Retinohypothalamic tract in the female albino rat: a study using horseradish peroxidase conjugated to cholera toxin. J Comp Neurol 306:344-360.

Liang FQ, Aleman TS, Dejneka NS, Dudus L, Fisher KJ, Maguire AM, Jacobson SG, Bennett J (2001) Long-term protection of retinal structure but not function using RAAV. CNTF in animal models of retinitis pigmentosa. Mol Ther 4:461-472.

Lu J, Shiromani P, Saper CB (1999) Retinal input to the sleep-active ventrolateral preoptic nucleus in the rat. Neuroscience 93:209-214.

Lu J, Greco MA, Shiromani P, Saper CB (2000a) Effect of lesions of the ventrolateral preoptic nucleus on NREM and REM sleep. J Neurosci 20:3830-3842.

Lu J, Zhang YH, Chou TC, Gaus SE, Elmquist JK, Shiromani P, Saper CB (2001) Contrasting effects of ibotenate lesions of the paraventricular nucleus and subparaventricular zone on sleep-wake cycle and temperature regulation. J Neurosci 21:4864-4874.

Lu J, Bjorkum AA, Xu M, Gaus SE, Shiromani PJ, Saper CB (2002) Selective activation of the extended ventrolateral preoptic nucleus during rapid eye movement sleep. J Neurosci 22:4568-4576.

Lucas RJ, Freedman MS, Munoz M, Garcia-Fernandez JM, Foster RG (1999) Regulation of the mammalian pineal by non-rod, non-cone, ocular photoreceptors. Science 284:505-507.

Lucas RJ, Douglas RH, Foster RG (2001) Characterization of an ocular photopigment capable of driving pupillary constriction in mice. Nat Neurosci 4:621-626.

Lucas RJ, Hattar S, Takao M, Berson DM, Foster RG, Yau KW (2003) Diminished pupillary light reflex at high irradiances in melanopsinknockout mice. Science 299:245-247.

Martin KR, Klein RL, Quigley HA (2002) Gene delivery to the eye using adeno-associated viral vectors. Methods 28:267-275.

Miyamoto Y, Sancar A (1998) Vitamin B2-based blue-light photoreceptors in the retinohypothalamic tract as the photoactive pigments for setting the circadian clock in mammals. Proc Natl Acad Sci USA 95:6097-6102.

Moga MM, Moore RY (1997) Organization of neural inputs to the suprachiasmatic nucleus in the rat. J Comp Neurol 389:508-534.

Moore RY, Weis R, Moga MM (2000) Efferent projections of the intergeniculate leaflet and the ventral lateral geniculate nucleus in the rat J Comp Neurol 420:398-418.

Morin LP (1994) The circadian visual system. Brain Res Brain Res Rev 19:102-127.

Mrosovsky N (1994) In praise of masking: behavioural responses of retinally degenerate mice to dim light. Chronobiol Int 11:343-348.

Mrosovsky N (1996) Locomotor activity and non-photic influences on circadian clocks. Biol Rev Camb Philos Soc 71:343-372.

Mrosovsky N (2001) Further characterization of the phenotype of mCry1/ mCry2-deficient mice. Chronobiol Int 18:613-625.

Mrosovsky N, Foster RG, Salmon PA (1999) Thresholds for masking responses to light in three strains of retinally degenerate mice. J Comp Physiol [A] 184:423-428.

Mrosovsky N, Lucas RJ, Foster RG (2001) Persistence of masking responses to light in mice lacking rods and cones. J Biol Rhythms 16:585-588.

Novak CM, Nunez AA (2000) A sparse projection from the suprachiasmatic nucleus to the sleep active ventrolateral preoptic area in the rat. NeuroReport 11:93-96.

Panda S, Sato TK, Castrucci AM, Rollag MD, DeGrip WJ, Hogenesch JB, Provencio I, Kay SA (2002) Melanopsin (Opn4) requirement for normal light-induced circadian phase shifting. Science 298:2213-2216.

Paxinos G, Watson C (1997) The rat brain in stereotaxic coordinates. San Diego: Academic.

Pickard GE (1985) Bifurcating axons of retinal ganglion cells terminate in the hypothalamic suprachiasmatic nucleus and the intergeniculate leaflet of the thalamus. Neurosci Lett 55:211-217.

Provencio I, Foster RG (1995) Circadian rhythms in mice can be regulated by photoreceptors with cone-like characteristics. Brain Res 694:183-190.

Provencio I, Rodriguez IR, Jiang G, Hayes WP, Moreira EF, Rollag MD (2000) A novel human opsin in the inner retina. J Neurosci 20:600-605.

Provencio I, Rollag MD, Castrucci AM (2002) Photoreceptive net in the mammalian retina. Nature 415:493.

Redlin U, Mrosovsky N (1999) Masking by light in hamsters with SCN lesions. J Comp Physiol [A] 184:439-448.

Ruby NF, Brennan TJ, Xie X, Cao V, Franken P, Heller HC, O’Hara BF (2002) Role of melanopsin in circadian responses to light. Science 298:2211-2213.

Sherin JE, Shiromani PJ, McCarley RW, Saper CB (1996) Activation of ventrolateral preoptic neurons during sleep. Science 271:216-219.

Sherin JE, Elmquist JK, Torrealba F, Saper CB (1998) Innervation of histaminergic tuberomammillary neurons by GABAergic and galaninergic neurons in the ventrolateral preoptic nucleus of the rat. J Neurosci $18: 4705-4721$ 
Takahashi JS, DeCoursey PJ, Bauman L, Menaker M (1984) Spectral sensitivity of a novel photoreceptive system mediating entrainment of mammalian circadian rhythms. Nature 308:186-188.

Thapan K, Arendt J, Skene DJ (2001) An action spectrum for melatonin suppression: evidence for a novel non-rod, non-cone photoreceptor system in humans. J Physiol (Lond) 535:261-267.

Van Gelder RN, Wee R, Lee JA, Tu DC (2003) Reduced pupillary light responses in mice lacking cryptochromes. Science 299:222.

Watts AG (1991) The efferent projections of the suprachiasmatic nucleus: anatomical insights into the control of circadian rhythms. In: Suprachiasmatic nucleus (Klein DC, Moore RY, Reppert SM, eds), pp 77-106. New York: Oxford UP.
Watts AG, Swanson LW, Sanchez-Watts G (1987) Efferent projections of the suprachiasmatic nucleus. I. Studies using anterograde transport of Phaselous vulgaris leucoagglutinin in rat. J Comp Neurol 258:204-229.

Yoshimura T, Ebihara S (1996) Spectral sensitivity of photoreceptors mediating phase-shifts of circadian rhythms in retinally degenerate $\mathrm{CBA} / \mathrm{J}(\mathrm{rd} / \mathrm{rd})$ and normal CBA/N $(+/+)$ mice. J Comp Physiol [A] 178:797-802.

Young MJ, Lund RD (1994) The anatomical substrates subserving the pupillary light reflex in rats: origin of the consensual pupillary response Neuroscience 62:481-496.

Young MJ, Lund RD (1998) The retinal ganglion cells that drive the pupilloconstrictor response in rats. Brain Res 787:191-202. 